\title{
A holographic proof of Rényi entropic inequalities
}

\author{
Yuki Nakaguchi ${ }^{a, b}$ and Tatsuma Nishioka ${ }^{b}$ \\ ${ }^{a}$ Kavli Institute for the Physics and Mathematics of the Universe, The University of Tokyo, \\ 5-1-5 Kashiwa-no-Ha, Kashiwa City, Chiba 277-8568, Japan \\ ${ }^{b}$ Department of Physics, Faculty of Science, The University of Tokyo, \\ Bunkyo-ku, Tokyo 113-0033, Japan \\ E-mail: yuuki.nakaguchi@ipmu.jp, nishioka@hep-th.phys.s.u-tokyo.ac.jp
}

\begin{abstract}
We prove Rényi entropic inequalities in a holographic setup based on the recent proposal for the holographic formula of Rényi entropies when the bulk is stable against any perturbation. Regarding the Rényi parameter as an inverse temperature, we reformulate the entropies in analogy with statistical mechanics, which provides us a concise interpretation of the inequalities as the positivities of entropy, energy and heat capacity. This analogy also makes clear a thermodynamic structure in deriving the holographic formula. As a by-product of the proof we obtain a holographic formula to calculate the quantum fluctuation of the modular Hamiltonian. A few examples of the capacity of entanglement are examined in detail.
\end{abstract}

KEYwORDS: AdS-CFT Correspondence, Gauge-gravity correspondence

ARXIV EPRINT: 1606.08443 


\section{Contents}

1 Introduction 1

2 Analogy to statistical mechanics 4

2.1 Partition function $Z$ and the escort density matrix $\rho_{n} \quad 4$

2.2 Improved Rényi entropy $\tilde{S}_{n}$

2.3 Capacity of entanglement $C(n) \quad 6$

2.4 Rényi entropic inequalities from the viewpoint of the analogy 6

3 Holographic formula of the Rényi entropy 7

3.1 The area prescription $\quad 7$

3.2 Derivation revisited from the viewpoint of the analogy 8

4 Proof of the Rényi entropic inequalities $\quad 11$

$\begin{array}{ll}4.1 \text { A holographic proof } & 11\end{array}$

4.2 Legendre transformed expression for capacity of entanglement 12

5 Calculations of the capacity of entanglement $\quad 14$

$\begin{array}{ll}5.1 \text { Conformal field theory } & 14\end{array}$

$\begin{array}{lll}5.2 \text { Free fields } & 15\end{array}$

$\begin{array}{ll}5.3 \text { Gravity duals } & 17\end{array}$

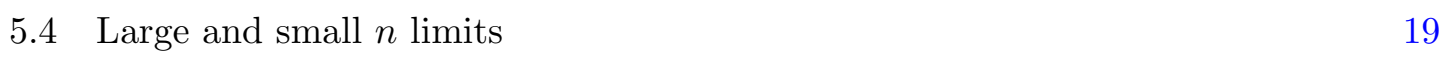

6 Discussion $\quad 20$

A On holographic calculation of $C(1)$ using graviton propagator $\quad 22$

B Comments on the strong sub-additivity of Rényi entropies 24

\section{Introduction}

A key concept in modern quantum gravity theory is holography that opened the door to the non-perturbative definition as the dual quantum theory in one lower dimensions. A considerable number of dictionaries have been composed to translate physical quantities in one theory to the other. The holographic duality remains as mysterious as quantum gravity, though, especially on how the bulk spacetime information is encoded in the boundary quantum field theory. There have been a huge amount of attempts to probe the bulk structure via holography, of which one of the most important breakthroughs is the holographic formula of entanglement entropy [1] associating a unit area per four times the Planck length of a codimension-two bulk surface with one bit of information for a given 
region in the boundary field theory. In fact, the formula is a realization of the original idea of the holographic principle $[2,3]$ that states in quantum gravity theory, the degrees of freedom live not in volumes but in areas. Overviews on the recent developments of the holographic entanglement entropy are available in reviews e.g. [4, 5].

In quantum theories, entanglement entropy $S_{A}$ of a state subspace $\mathcal{H}_{A}$ is defined as the von Neumann entropy $S_{\mathrm{vN}}[\rho] \equiv-\operatorname{Tr}[\rho \log \rho]$ of the reduced matrix $\rho_{A}=\operatorname{Tr}_{\bar{A}}\left[\rho_{\text {total }}\right]$ as $^{1}$

$$
S_{A} \equiv-\operatorname{Tr}\left[\rho_{A} \log \rho_{A}\right] .
$$

It measures how much quantum information of the degrees of freedom in $\mathcal{H}_{A}$ is entangled with the outer degrees of freedom, namely, how much quantum information will be lost for the subspace $\mathcal{H}_{A}$ if the outer subspace is ignored. In quantum field theories, entanglement entropy is defined for a space region $A$ on a time slice, assuming that we can construct a state space $\mathcal{H}_{A}$ representing degrees of freedom on the region $A$ by some appropriate procedures. The total state is often taken as the vacuum $\rho_{\text {total }}=|0\rangle\langle 0|$ for simplicity.

Entanglement entropy has many mathematical properties, among which the most important one is an inequality called the strong sub-additivity [6]

$$
S_{A C}+S_{B C} \geq S_{C}+S_{A B C},
$$

showing a kind of concavity of the entropy. The sub-additivity

$$
S_{A}+S_{B} \geq S_{A B}
$$

follows by taking $C$ as $\emptyset$. As a field application, the strong sub-additivity is utilized for constructing $c$-functions, monotonically decreasing functions along RG flows, such as the entropic $c$-function in two dimensions [7] and the $F$-function in three dimensions [8].

One of the novel aspects of the holographic entanglement entropy formula is the simplicity of proving the strong sub-additivity (1.2) [9-11]. The proof only relies on the geometric properties of a codimension-two surface in the bulk, and suggests a profound way of the emergence of the bulk spacetime as it translates a quantum mechanical constraint to a purely geometric one. More extensive studies of the inequalities satisfied by the holographic formula were carried out in $[12,13]$ to classify the characteristics of the geometry which has a field theory dual.

Recently, the holographic formula was proposed [14] for the entanglement Rényi entropy $S_{n}[\rho]$ which is a one-parameter generalization of the von Neumann entropy defined with a non-negative real number $n$ as

$$
S_{n}[\rho] \equiv-\frac{1}{n-1} \log \operatorname{Tr}\left[\rho^{n}\right] .
$$

It reduces to the von Neumann entropy when $n=1, S_{1}[\rho]=S_{\mathrm{vN}}[\rho]$. The derivation of the holographic formula by [14] is based on so-called the Lewkowycz-Maldacena prescription [15] employed to derive the holographic entanglement entropy where the replica $\mathbb{Z}_{n}$

\footnotetext{
${ }^{1}$ Throughout this paper, we always normalize a density matrix as $\operatorname{Tr}[\rho]=1$.
} 
symmetry is assumed in the bulk geometry. ${ }^{2}$ We will review the derivation in section 3 so as to fix our notations and for later use.

Then it is natural to think about how mathematical properties of the Rényi entropy are transcribed to the bulk side in a geometric language. It is known that the Rényi entropy is not strongly sub-additive, but it satisfies inequalities involving the derivative with respect to $n[19,20]^{3}$

$$
\begin{aligned}
\partial_{n} S_{n} & \leq 0, \\
\partial_{n}\left(\frac{n-1}{n} S_{n}\right) & \geq 0, \\
\partial_{n}\left((n-1) S_{n}\right) & \geq 0, \\
\partial_{n}^{2}\left((n-1) S_{n}\right) & \leq 0 .
\end{aligned}
$$

These inequalities are originally proved for the classical Rényi entropy $S_{n}\left[p_{i}\right] \equiv-\frac{1}{n-1} \sum_{i} p_{i}^{n}$ of a probability distribution $p_{i}$, but are still true for the quantum Rényi entropy (1.4). The proof for a quantum case immediately follows by diagonalizing the density matrix $\rho$ as $U \rho U^{\dagger}=\operatorname{diag}\left(p_{1}, p_{2}, \ldots\right)$ with a unitary matrix $U$. The first inequality (1.5) implies the positivity of the Rényi entropy $S_{n} \geq 0$ as $S_{\infty}=\min _{i}\left(-\log p_{i}\right) \geq 0$.

The aim of this paper is to prove these inequalities by the holographic formula of the Rényi entropy. Before proceeding to the proof, we rewrite the inequalities in more concise forms that manifest their meanings as the positivities of energy, entropy and heat capacity in analogy to statistical mechanics. It also clarifies that not all of (1.5)-(1.8) are independent, but the two inequalities (1.6) and (1.8) are essential. (1.6) turns out to be simple to prove as it stands for the positivity of the area of a codimension-two surface in the bulk, while the proof of (1.8) is more intricate. In view of statistical mechanics, (1.8) implies the positivity of the heat capacity and encodes the unitarity of quantum mechanical system. Our proof of (1.8) in the bulk is differential geometric in its nature and turns out to relate it to the stability of the spacetime on which the holographic formula is supposed to be applied. Therefore, our proof serves as a nontrivial consistency check for the holographic formula, and moreover reveals a direct connection between the unitarity and the stability in the boundary and bulk theories, respectively. In due course of the proof, we also obtain a holographic formula for calculating the quantum fluctuation of the modular Hamiltonian.

Our proof is heavily based on the stability of the bulk geometry. We admit that the bulk stability is a nontrivial assumption whose justification is even challenging. For instance, Euclidean gravity actions are known to be indefinite against metric perturbations [21, 22]. We are not aware of any compelling argument to support the assumption, but in view of holographic duality we believe that stable quantum states should have stable bulk duals. We will not touch on this subject anymore in this paper until section 6 .

The organization of this paper is as follows. In section 2 we reformulate the Rényi entropy and its inequalities in a way analogous to statistical mechanics and introduce a

\footnotetext{
${ }^{2}$ Earlier works on the holographic Rényi entropies include [16-18].

${ }^{3}$ The finite version of these inequalities, such as $S_{n} \geq S_{m}$ and $\frac{n-1}{n} S_{n} \geq \frac{m-1}{m} S_{m}$ for $n \leq m$, are true, even if the $n$ derivatives are ill-defined because of some discontinuity.
} 
notion of the heat capacity of entanglement. The holographic formula of the Rényi entropy is reviewed in section 3 with emphasis on the analogy to statistical mechanics. In section 4 , we prove the Rényi entropic inequalities from the holographic point of view. The capacity of entanglement is exemplified in various systems in section 5. Finally section 6 is devoted to discussions on our results and future directions. Appendix A deals with an alternative method of computing the capacity of entanglement in the holographic setup and discusses a delicate issue arising from boundary terms. A possible counterpart of the strong subadditivity for the Rényi entropy is elaborated in appendix B.

\section{$2 \quad$ Analogy to statistical mechanics}

The Rényi entropy can be recasted as a thermal entropy when the region $A$ is a ball in $\mathrm{CFT}_{d}$ as the replica manifold $\mathcal{M}_{n}$ is conformally equivalent to a thermal hyperbolic space $\mathbb{S}^{1} \times \mathbb{H}^{d-1}$ with an inverse temperature $\beta=2 \pi n[18,23]$. In that situation, the inequalities (1.6) and (1.8) reduce to the non-negativity of the thermal entropy and the heat capacity, and the others immediately follow from these two. A formal similarity between the Rényi entropy and a thermal entropy is also pointed out in [14].

In this section, inspired by these observations, we will formulate the complete analogy between the Rényi entropy and statistical mechanics valid for any quantum system. Moreover, the following discussions apply not only to reduced density matrices $\rho_{A}=\operatorname{Tr}_{\bar{A}}\left[\rho_{\text {tot }}\right]$, but also to a general density matrix $\rho$.

\subsection{Partition function $Z$ and the escort density matrix $\rho_{n}$}

In the calculation of the Rényi entropy $S_{n}=-\frac{1}{n-1} \log \operatorname{Tr}\left[\rho^{n}\right]$ (1.4), we can regard the trace $Z(n) \equiv \operatorname{Tr}\left[\rho^{n}\right]$ as a thermal partition function

$$
Z(\beta)=\operatorname{Tr}\left[e^{-\beta H}\right]
$$

with $^{4}$ the inverse temperature $\beta$ and the Hamiltonian $H$

$$
\begin{aligned}
\beta & =n, \\
H & =-\log \rho .
\end{aligned}
$$

The latter is called the entanglement Hamiltonian or modular Hamiltonian. Its eigenvalues $\epsilon_{i}$ are called the entanglement spectrum, and are non-negative $\epsilon_{i} \geq 0$ as the eigenvalues $p_{i}=e^{-\epsilon_{i}}$ of $\rho$ satisfies $0 \leq p_{i} \leq 1$. In calculating the partition function $Z$, we can regard the state as a density matrix given by the normalized $n$-th power of $\rho$

$$
\rho_{n} \equiv \frac{\rho^{n}}{\operatorname{Tr}\left[\rho^{n}\right]} .
$$

\footnotetext{
${ }^{4}$ If you feel uneasy about the mismatch of their physical dimensions, you may define them instead as

$$
\begin{aligned}
\beta E_{0} & =n, \\
H / E_{0} & =-\log \rho,
\end{aligned}
$$

with any constant $E_{0}$ of the dimension of energy. In the following discussions we take a unit $E_{0}=1$. Another choice $E_{0}=1 / 2 \pi$ is also common in literatures.
} 
In the area of chaotic systems, the probability distribution of the classical version $P_{i}^{(n)} \equiv$ $p_{i}^{n} / \sum_{i} p_{i}^{n}$ is called the escort distribution [20], and we will accordingly call $\rho_{n}$ the escort density matrix.

Let us push forward this analogy to statistical mechanics. The free energy $F=F(n)$ and the total energy $E=E(n)$ related to the density matrix $\rho$ are defined as

$$
\begin{aligned}
& F \equiv-\frac{1}{n} \log \operatorname{Tr}\left[\rho^{n}\right], \\
& E \equiv-\frac{\partial}{\partial n} \log \operatorname{Tr}\left[\rho^{n}\right]=\langle H\rangle_{n},
\end{aligned}
$$

where $\langle\cdot\rangle_{n}$ stands for the expectation values with respect to the escort density matrix $\rho_{n}$,

$$
\langle X\rangle_{n} \equiv \operatorname{Tr}\left[\rho_{n} X\right]=\frac{\operatorname{Tr}\left[\rho^{n} X\right]}{\operatorname{Tr}\left[\rho^{n}\right]} .
$$

In what follows, we will make use of this notation when available.

\subsection{Improved Rényi entropy $\tilde{\boldsymbol{S}}_{n}$}

What quantity should correspond to the thermal entropy in this analogy to statistical mechanics? The answer is not the Rényi entropy $S_{n}[\rho]$, but a more involved function:

$$
\begin{aligned}
\tilde{S}_{n}[\rho] & \equiv n^{2} \partial_{n}\left(\frac{n-1}{n} S_{n}\right), \\
& =\left(1-n \partial_{n}\right) \log \operatorname{Tr}\left[\rho^{n}\right], \\
& =\partial_{1 / n}\left(\frac{1}{n} \log \operatorname{Tr}\left[\rho^{n}\right]\right) .
\end{aligned}
$$

Let us call this $\tilde{S}_{n}[\rho]$ as the improved Rényi entropy. In fact, the equation (2.11) or (2.12) leads to the formulae of the entropy together with (2.7) and (2.8),

$$
\begin{aligned}
\tilde{S} & =n(E-F), \\
& =-\frac{\partial F}{\partial T},
\end{aligned}
$$

where $T \equiv 1 / n$ and we omit the subscript of $\tilde{S}_{n}$ to stress the correspondence to statistical mechanics. One can also show that the improved Rényi entropy is nothing but the von Neumann entropy of the escort density matrix $\rho_{n}$, that is,

$$
\tilde{S}_{n}[\rho]=S_{\mathrm{vN}}\left[\rho^{n} / \operatorname{Tr}\left[\rho^{n}\right]\right] .
$$

The improved Rényi entropy $\tilde{S}_{n}$ is another generalization of the von Neumann entropy $S_{\mathrm{vN}}$ as it also reduces to the entanglement entropy $\tilde{S}_{1}[\rho]=S_{\mathrm{vN}}[\rho]$ in the limit $n \rightarrow 1$.

An equivalent relation to $(2.10)$

$$
(n-1)^{2} \partial_{n} S_{n}=\tilde{S}_{n}-E,
$$

yields a useful formula for calculating $\partial_{n} S_{n}$ in terms of $F$

$$
\partial_{n} S_{n}=T^{2} \frac{F(1)-F(T)-(1-T) \partial_{T} F}{(1-T)^{2}},
$$

where we used the relations $E=F+T \tilde{S}, \tilde{S}=-\partial_{T} F$ and $F(1)=0$. 


\subsection{Capacity of entanglement $C(n)$}

Now that we have defined thermodynamic state functions consisting of the first derivative of the free energy such as the total energy $E=\partial_{n}(n F)$ and the thermal entropy $\tilde{S}=-\partial_{T} F$, we proceed to implement the heat capacity $C=C(n)$ including the second derivative,

$$
C \equiv \frac{\partial E}{\partial T}=T \frac{\partial \tilde{S}}{\partial T}=-T \frac{\partial^{2} F}{\partial T^{2}}
$$

It was originally introduced to characterize topologically ordered states by [24] and named capacity of entanglement. The capacity of entanglement has not attracted much attention so far despite its importance and simplicity as we will see below.

One can show the non-negativity $C \geq 0$ as in the same way as statistical mechanics,

$$
\begin{aligned}
C(n)=n^{2} \frac{\partial^{2}}{\partial n^{2}} \log Z(n) & =n^{2}\left(\left\langle H^{2}\right\rangle_{n}-\langle H\rangle_{n}^{2}\right), \\
& =n^{2}\left\langle\left(H-\langle H\rangle_{n}\right)^{2}\right\rangle_{n} .
\end{aligned}
$$

It follows that the capacity measures the quantum fluctuation of the modular Hamiltonian $H=-\log \rho$, and in particular $C(1)=\left\langle H^{2}\right\rangle-\langle H\rangle^{2}$ gives the quantum fluctuation with respect to the original state $\rho$.

\subsection{Rényi entropic inequalities from the viewpoint of the analogy}

Having established the analogy to statistical mechanics, we rewrite the Rényi entropic inequalities in the thermodynamic representation. The second (1.6), third (1.7) and forth (1.8) inequalities turn out to be the non-negativity of the improved Rényi entropy $\tilde{S}$ (2.10), the total energy $E(2.8)$ and the entanglement heat capacity $C$, respectively

$$
\begin{aligned}
& \tilde{S} \geq 0, \\
& E \geq 0, \\
& C \geq 0 .
\end{aligned}
$$

The non-negativity of $\tilde{S}$ and $E=\langle H\rangle_{n}$ immediately follows from the relations $\tilde{S}_{n}[\rho]=$ $\left\langle-\log \rho_{n}\right\rangle_{n}=S_{\mathrm{vN}}\left[\rho_{n}\right]$ and the definition $H=-\log \rho$ of the modular hamiltonian. The last inequality $C \geq 0$ has already been proved by (2.18). Note that the condition $C \geq 0$ is equivalent to

$$
\partial_{n} \tilde{S}_{n} \leq 0
$$

because of $C=T \partial_{T} \tilde{S}=-n \partial_{n} \tilde{S}$.

The first inequality $\partial_{n} S_{n} \leq 0(1.5)$ can be derived from the forth inequality (1.8) as shown in [18]. Indeed, the forth inequality $C=-T \partial_{T}^{2} F \geq 0$ is equivalent to the concavity of the free energy $F$, and (2.16) is clearly non-positive as $f(x) \leq f(a)+(x-a) f^{\prime}(a)$ for any concave function $f(x)$. An alternative way to show this inequality uses the non-negativity of the relative entropy $S[\rho \mid \sigma] \equiv \operatorname{Tr}[\rho(\log \rho-\log \sigma)] \geq 0$ for $(2.15)$

$$
\begin{aligned}
(n-1)^{2} \partial_{n} S_{n} & =\tilde{S}_{n}-E, \\
& =-\left\langle\log \rho_{n}-\log \rho\right\rangle_{n}, \\
& =-S\left[\rho_{n} \mid \rho\right] \leq 0 .
\end{aligned}
$$




\section{Holographic formula of the Rényi entropy}

We review the holographic formula for the Rényi entropy and its derivation proposed by [14], with some clarifications on the thermodynamic interpretation developed in the previous section. It resembles to the Ryu-Takayanagi formula, but is more intricate as the entropy is given by the area of a cosmic brane with a tension depending on the parameter $n$, which is extremized in the backreacted geometry. The derivation of the formula still proceeds along with the Lewkowycz-Maldacena prescription [15].

\subsection{The area prescription}

The holographic formula for the Rényi entropy [14] states that the improved Rényi entropy $\tilde{S}_{n}$ of a region $A$ in $\mathrm{QFT}_{d}$ is given by the area $\mathcal{A}$ of a codimension-two surface $C_{A}^{(n)}$ in an asymptotically $\mathrm{AdS}_{d+1}$ space as

$$
\tilde{S}_{n}=\left.\frac{\mathcal{A}}{4 G_{N}}\right|_{\delta I=0, \partial C_{A}^{(n)}=\partial A},
$$

where the surface $C_{A}^{(n)}$ is anchored on $\partial A$ on the asymptotic boundary of the bulk spacetime. Unlike the Ryu-Takayanagi formula, the surface $C_{A}^{(n)}$ is to be fixed by minimizing an $n$-dependent Euclidean action $I=I_{\text {bulk }}+I_{\text {brane }}$. Here $I_{\text {bulk }}$ is the original bulk action in the dual gravity theory consisting of the Einstein-Hilbert action, the cosmological constant term and matter terms

$$
I_{\mathrm{bulk}}\left[G_{\mu \nu}(X), \psi(X)\right]=I_{\mathrm{EH}}\left[G_{\mu \nu}(X)\right]+I_{\Lambda}\left[G_{\mu \nu}(X)\right]+I_{\text {matters }}\left[G_{\mu \nu}(X), \psi(X)\right],
$$

where $G_{\mu \nu}(X)$ the bulk metric, $\psi(X)$ matter fields, and $X^{\mu}(\mu=0, \ldots, d)$ is the bulk coordinate. If we extremize the codimension-two surface with this bulk action, we end up with the Ryu-Takayanagi surface for the holographic entanglement entropy. A new ingredient of the prescription for the Rényi entropy is a cosmic brane action $I_{\text {brane }}$ of $C_{A}^{(n)}$,

$$
I_{\text {brane }}\left[G_{\mu \nu}(X), X^{\mu}(y)\right]=T_{n} \mathcal{A}\left[G_{\mu \nu}(X), X^{\mu}(y)\right],
$$

which is just the product of a brane tension $T_{n}$ given by

$$
T_{n}=\frac{1}{4 G_{N}} \frac{n-1}{n},
$$

and the area of the surface $C_{A}^{(n)}$

$$
\mathcal{A}=\int_{C_{A}^{(n)}} d^{d-1} y \sqrt{g(y)} .
$$

Here $X^{\mu}(y)$ specify the embedding of the surface into the bulk, $y^{i}(i=1, \ldots, d-1)$ the embedding coordinate, and $g_{i j}(y)$ the induced metric on $C_{n}$,

$$
g_{i j}(y)=G_{\mu \nu}(X(y)) \frac{\partial X^{\mu}}{\partial y^{i}} \frac{\partial X^{\nu}}{\partial y^{j}} .
$$


The main difference from the Ryu-Takayanagi formula arises from the back-reaction of the codimension-two surface to the bulk metric $G_{\mu \nu}$. Namely we extremize the action including the cosmic brane:

$$
0=\frac{\delta I}{\delta G_{\mu \nu}(X)}=\frac{\delta I_{\text {bulk }}}{\delta G_{\mu \nu}(X)}+T_{n} \frac{\delta \mathcal{A}}{\delta G_{\mu \nu}(X)},
$$

where the first term is the original bulk equation of motion, and the second term is essentially the energy-momentum tensor of the cosmic brane $C_{A}^{(n)}$. Note that $C_{A}^{(n)}$ is still a minimal surface as the equation of motion for the embedding $X^{\mu}(y)$ shows:

$$
\frac{\delta I}{\delta X^{\mu}(y)}=T_{n} \frac{\delta \mathcal{A}}{\delta X^{\mu}(y)}=0 .
$$

This equation should be evaluated on the backreacted bulk metric $G_{\mu \nu}$. When there are matter fields $\psi$, we also have to solve

$$
\frac{\delta I}{\delta \psi}=\frac{\delta I_{\text {matters }}}{\delta \psi}=0
$$

in the backreacted background $G_{\mu \nu}$. The Ryu-Takayanagi formula

$$
S_{A}=\min _{\partial C_{A}=\partial A} \frac{\mathcal{A}\left[C_{A}\right]}{4 G_{N}},
$$

is recovered from (3.1) in the limit $n \rightarrow 1$ where the brane tension $T_{n}$ vanishes and we can neglect the backreaction of the brane.

\subsection{Derivation revisited from the viewpoint of the analogy}

To derive the holographic formula (3.1), we employ the replica trick relating the trace of the density $\rho$ to the Euclidean partition function $Z$ [25],

$$
\log \operatorname{Tr}\left[\rho^{n}\right]=\log Z\left[M_{n}\right]-n \log Z\left[M_{1}\right],
$$

where $M_{n}$ is the $n$-fold cover branched over the region $A$. In the classical gravity regime, there exists a regular solution $B_{n}$ of the Einstein equation holographically dual to the field theory on the replica manifold $M_{n}$ such that $\partial B_{n}=M_{n}$. The partition function $Z$ is equated to the on-shell bulk action on $B_{n}$ :

$$
Z\left[M_{n}\right]=Z_{\text {bulk }} \sim e^{-I_{\text {bulk }}\left[B_{n}\right]} .
$$

$n$ has been supposed to be an integer up to now, but we analytically continue it to an arbitrary real number. Such an analytic continuation can be performed in the bulk side by defining the "bulk per replica" manifold

$$
\hat{B}_{n}=B_{n} / \mathbb{Z}_{n},
$$

under the assumption that the replica symmetry $\mathbb{Z}_{n}$ extends to the on-shell bulk solution $B_{n}[15] .^{5}$ This quotient geometry $\hat{B}_{n}$ has a conical singularity at a codimension-two fixed locus $C_{A}^{(n)}$ of the $\mathbb{Z}_{n}$ symmetry with a deficit angle

$$
\Delta \phi=2 \pi(1-1 / n) .
$$

\footnotetext{
${ }^{5}$ See $[26]$ for the discussion on the replica symmetry $\mathbb{Z}_{n}$ breaking.
} 
The fixed locus $C_{A}^{(n)}$ extends to the AdS boundary and touches on the entangling surface $\partial A$ which is also fixed locus of the replica symmetry.

Next, let us define "bulk action per replica" $I$ for the quotient $\hat{B}_{n}$, just by dividing the bulk on-shell action $I_{\text {bulk }}\left[B_{n}\right]$ by $n$,

$$
I \equiv I_{\mathrm{bulk}}\left[B_{n}\right] / n
$$

This action $I$ differs from $I_{\text {bulk }}\left[\hat{B}_{n}\right]$ of the quotient bulk $\hat{B}_{n}$, and has an additional contribution from the singularity at $C_{A}^{(n)} \cdot{ }^{6}$ Bearing in mind that $\hat{B}_{n}$ is locally the same as the original bulk $B_{n}$ away from the conical singularity $C_{A}^{(n)}$, the Ricci scalar $R(X)$ of $\hat{B}_{n}$ takes the following form $[27]$

$$
\left.\sqrt{G(X)} R(X)\right|_{\hat{B}_{n}}=\left.\sqrt{G(X)} R(X)\right|_{B_{n}}+2 \Delta \phi \int_{C_{A}^{(n)}} d^{d-1} y \sqrt{g} \delta^{d+1}(X-X(y)) .
$$

Thus in the Einstein gravity $I_{\mathrm{EH}}=-\frac{1}{16 \pi G_{N}} \int d^{d+1} X \sqrt{G(X)} R(X)$,

$$
\begin{aligned}
I_{\text {bulk }}\left[\hat{B}_{n}\right] & =I_{\text {bulk }}\left[B_{n}\right] / n-\frac{\Delta \phi}{8 \pi G_{N}} \int_{C_{A}^{(n)}} d^{d-1} y \sqrt{g}, \\
& =I-\frac{1-1 / n}{4 G_{N}} \mathcal{A},
\end{aligned}
$$

which means that the action $I$ includes the area term

$$
I=I_{\mathrm{bulk}}\left[\hat{B}_{n}\right]+T_{n} \mathcal{A},
$$

with the correct brane tension (3.4)

$$
T_{n}=\frac{\Delta \phi}{8 \pi G_{N}}=\frac{1-1 / n}{4 G_{N}},
$$

and the area $\mathcal{A}(3.5)$ as desired.

A point of caution is that not $I_{\text {bulk }}\left[\hat{B}_{n}\right]$ itself, but the combination $I=I_{\text {bulk }}\left[\hat{B}_{n}\right]+$ $T_{n} \mathcal{A}$ is on-shell with respect to the bulk fields $G_{\mu \nu}(X)$ and $\psi(X)$. This is clear from the relation (3.15) and $B_{n}$ being the regular solution for the action $I_{\mathrm{bulk}}\left[B_{n}\right]$.

The replica symmetry would constrain the embedding $X^{\mu}(y)$ to be the minimal surface $\delta \mathcal{A} / \delta X^{\mu}(y)=0 .{ }^{7}$ We promote the embedding $X^{\mu}(y)$ to a dynamical variable and minimize the action $I$ with respect to $X^{\mu}(y)$ in order to analytically continue $n$ to a real number.

\footnotetext{
${ }^{6}$ Here our notation of $I_{\text {bulk }}\left[\hat{B}_{n}\right]$ is different from that in other literatures such as [14]. Our $I_{\text {bulk }}\left[\hat{B}_{n}\right]$ includes the contribution from the conical singularity $C_{A}^{(n)}$, while their $I_{\mathrm{bulk}}\left[\hat{B}_{n}\right]$ means $I_{\mathrm{bulk}}\left[\hat{B}_{n} \backslash C_{A}^{(n)}\right]=$ $I_{\text {bulk }}\left[B_{n}\right] / n=I$ without the contribution from $C_{A}^{(n)}$.

${ }^{7}$ We could justify this statement somewhat by a following rough argument. Consider how the area $\mathcal{A}$ would change in the leading order of a perturbation $\epsilon^{\mu}(y)$ of the embedding $X^{\mu}(y)$, in the bulk $\hat{B}_{n}$. In the original bulk $B_{n}$, where $n$ copies of $\hat{B}_{n}$ are glued at the surface, let us call the vector $\epsilon^{\mu}(y)$ toward the $i$-th copy of $\hat{B}_{n}$ as $\epsilon_{i}^{\mu}(y)$. Since the original surface $X^{\mu}(y)$ is invariant under the replica $\mathbb{Z}_{n}$ symmetry shifting $\epsilon_{i}^{\mu}(y)$ to $\epsilon_{i+1}^{\mu}(y)$, the variation of the area $\frac{\delta \mathcal{A}}{\delta X^{\mu}} \epsilon_{i}^{\mu}$ does not depend on the label $i$ and in fact $\frac{\delta \mathcal{A}}{\delta X^{\mu}} \epsilon_{i}^{\mu}=\frac{\delta \mathcal{A}}{\delta X^{\mu}} \epsilon^{\mu}$. On the other hand, the sum of these vectors vanishes $\sum_{i=1}^{n} \epsilon_{i}^{\mu}=0$ because of the symmetry. Then $0=\frac{\delta \mathcal{A}}{\delta X^{\mu}} \sum_{i=1}^{n} \epsilon_{i}^{\mu}=n \frac{\delta \mathcal{A}}{\delta X^{\mu}} \epsilon^{\mu}$ means that the area is minimal $\frac{\delta \mathcal{A}}{\delta X^{\mu}}=0$.
} 
Combining the replica trick (3.11) and the holographic relation (3.12) together with the definition of the action $I$ (3.15), we have the expression

$$
\begin{aligned}
\log \operatorname{Tr}\left[\rho^{n}\right] & =-\left(I_{\text {bulk }}\left[B_{n}\right]-n I_{\text {bulk }}\left[B_{1}\right]\right) \\
& =-n\left(I-\left.I\right|_{n=1}\right)
\end{aligned}
$$

from which the free energy $F(T)$ follows as the difference of the actions between $n$ and $n=1$

$$
F=-\frac{1}{n} \log \operatorname{Tr}\left[\rho^{n}\right]=I-\left.I\right|_{n=1} .
$$

The second term $-\left.I\right|_{n=1}$ ensures the normalization $F(1)=-\log \operatorname{Tr}[\rho]=0$. The free energy results from the minimization with respect to the fields $\phi=\left\{G_{\mu \nu}(X), \psi(X), X^{\mu}(y)\right\}$

$$
F(T)=\min _{\phi}(I[\phi])-\left.I\right|_{n=1},
$$

as the action $I$ is on-shell. Here we introduce a temperature $T=1 / n$ and rewrite the action as

$$
I=I_{\mathrm{bulk}}\left[\hat{B}_{n}\right]+(1-T) \frac{\mathcal{A}}{4 G_{N}} .
$$

This succinct form is convenient to derive the entropy $\tilde{S}_{n}$

$$
\tilde{S}_{n}=-\frac{\partial F}{\partial T}=-\frac{\delta I[\phi]}{\delta \phi} \frac{\delta \phi}{\delta T}+\frac{\mathcal{A}}{4 G_{N}}
$$

where the first and second terms come from the variation of the fields $\phi$ and the tension $T_{n}=(1-T) / 4 G_{N}$, respectively. Imposing the equations of motion, the first term vanishes $\delta I / \delta \phi=0$, and we reach the holographic Rényi entropy formula (3.1)

$$
\tilde{S}_{n}=\frac{\mathcal{A}}{4 G_{N}} .
$$

The derivation explains why one has to take into account the backreaction of the cosmic brane to the geometry while extremizing the area.

Finally we derive the total energy $E$ by the Legendre transformation

$$
\begin{aligned}
E & =F+T \tilde{S}_{n}, \\
& =I_{\text {bulk }}\left[\hat{B}_{n}\right]-I_{\text {bulk }}\left[B_{1}\right]+\frac{\mathcal{A}}{4 G_{N}} .
\end{aligned}
$$

This derivation is exactly the same as the one in thermodynamics; $\delta E-T \delta S$ vanishes because of the minimization in the Legendre transformation $F(T) \equiv \min _{S}(E(S)-T S)$, yielding $\delta F=\delta(E-T S)=(\delta E-T \delta S)-S \delta T=-S \delta T$. In our derivation of the holographic formula, the minimization of the free energy leads to a first-law like relation $0=\delta_{\phi} I=$ $\delta_{\phi} E-T \delta_{\phi} \tilde{S}$. The only difference is the meaning of the variation; $\delta_{\phi}$ is taken with respect to fields $\phi$ in our case. 


\section{Proof of the Rényi entropic inequalities}

Having established the necessary tools in the preceding sections, we want to examine under what condition the holographic formula (3.1) satisfies the inequalities (1.5)-(1.8) of the Rényi entropy. Instead of dealing with the original ones, we prove the concise inequalities (2.19)-(2.21) whose physical meaning is more transparent. They imply the stability of the system in the thermodynamic language, which is translated to the stability of the gravity theory as we will see soon.

\subsection{A holographic proof}

Some of the Rényi entropic inequalities follow straightforwardly from the holographic formula $\tilde{S}_{n}=\mathcal{A} / 4 G_{N}$ (3.1). The second inequality $\tilde{S} \geq 0(2.19)$ is trivial as the area $\mathcal{A}$ is always non-negative. The non-negativity of the Rényi entropy $S_{n}=\frac{n}{n-1} F \geq 0$, which is equivalent to $F<0$ for $n<1$ and $F>0$ for $n>1$, also follows from $\partial_{n} F=\tilde{S}_{n} / n^{2} \geq 0$ and $F(1)=0$. The first inequality (1.5) descents from the forth inequality (2.21) as mentioned in section 2 .

Let us move on to the proof of the forth inequality (2.21)

$$
C=-n \frac{\partial \tilde{S}_{n}}{\partial n}=-\frac{n}{4 G_{N}} \frac{\delta \mathcal{A}}{\delta n} \geq 0 .
$$

As the parameter $n$ varies slightly by $\delta n$, the brane area $\mathcal{A}$ changes slightly by

$$
\begin{aligned}
\frac{\delta \mathcal{A}[G, X]}{\delta n} & =\int d^{d+1} X \frac{\delta \mathcal{A}}{\delta G_{\mu \nu}(X)} \frac{\delta G_{\mu \nu}(X)}{\delta n}+\int d^{d-1} y \frac{\delta \mathcal{A}}{\delta X^{\mu}(y)} \frac{\delta X^{\mu}(y)}{\delta n}, \\
& =\int d^{d+1} X \frac{\delta \mathcal{A}}{\delta G_{\mu \nu}(X)} \frac{\delta G_{\mu \nu}(X)}{\delta n}
\end{aligned}
$$

where we used the minimality condition $\delta \mathcal{A} / \delta X^{\mu}=0$ for the embedding in the second equality. Plugging this result into (4.1), we have

$$
C=-\frac{n}{4 G_{N}} \int d^{d+1} X \frac{\delta \mathcal{A}}{\delta G_{\mu \nu}(X)} \frac{\delta G_{\mu \nu}(X)}{\delta n} .
$$

The derivatives $\delta \mathcal{A} / \delta G_{\mu \nu}$ and $\delta G_{\mu \nu}(X) / \delta n$ are not independent due to the equation of motion of the bulk metric $G_{\mu \nu}$. The variation with respect to $n$ gives

$$
\frac{\delta I_{\mathrm{bulk}}}{\delta G_{\mu \nu}(X)}[G+\delta G, \psi+\delta \psi]+\left(T_{n}+\delta T_{n}\right) \frac{\delta \mathcal{A}}{\delta G_{\mu \nu}(X)}[G+\delta G, X+\delta X]=0
$$

or

$$
\frac{\delta I}{\delta G_{\mu \nu}(X)}[G+\delta G, X+\delta X, \psi+\delta \psi]+\frac{\delta n}{4 G_{N} n^{2}} \frac{\delta \mathcal{A}}{\delta G_{\mu \nu}(X)}[G, \psi, X]=0,
$$

where we used $\delta T_{n}=\delta n /\left(4 G_{N} n^{2}\right)$. In the leading order of $\delta n$, the difference from the original equation motion is

$$
\begin{aligned}
\int d^{d+1} X^{\prime}\left[\frac{\delta^{2} I}{\delta G_{\mu \nu}(X) \delta G_{\alpha \beta}\left(X^{\prime}\right)} \delta G_{\alpha \beta}\left(X^{\prime}\right)+\frac{\delta^{2} I}{\delta G_{\mu \nu}(X) \delta \psi\left(X^{\prime}\right)} \delta \psi\left(X^{\prime}\right)\right] \\
+\int d^{d-1} y \frac{\delta^{2} I}{\delta G_{\mu \nu}(X) \delta X^{\alpha}(y)} \delta X^{\alpha}(y)+\frac{\delta n}{4 G_{N} n^{2}} \frac{\delta \mathcal{A}}{\delta G_{\mu \nu}}=0 .
\end{aligned}
$$


This gives the following relation

$$
\frac{\delta \mathcal{A}}{\delta G_{\mu \nu}(X)}=-4 G_{N} n^{2} \int d^{d+1} X^{\prime} \frac{\delta^{2} I}{\delta G_{\mu \nu}(X) \delta G_{\alpha \beta}\left(X^{\prime}\right)} \frac{\delta G_{\alpha \beta}\left(X^{\prime}\right)}{\delta n},
$$

where we used the equations of motion $\delta I / \delta \psi=0$ and $\delta I / \delta X^{\mu}=0$. Plugging this $\delta A / \delta G$ into (4.3), finally we obtain a symmetric formula for the capacity of entanglement ${ }^{8}$

$$
C=n^{3} \int d^{d+1} X d^{d+1} X^{\prime} \frac{\delta G_{\mu \nu}(X)}{\delta n} \frac{\delta^{2} I}{\delta G_{\mu \nu}(X) \delta G_{\alpha \beta}\left(X^{\prime}\right)} \frac{\delta G_{\alpha \beta}\left(X^{\prime}\right)}{\delta n} .
$$

To prove the non-negativity of $C$, it is sufficient to show that the Hessian matrix $\frac{\delta^{2} I}{\delta G_{\mu \nu}(X) \delta G_{\alpha \beta}\left(X^{\prime}\right)}$ is non-negative definite on the on-shell bulk $G_{\mu \nu}$. This condition means that the bulk geometry is stable against any perturbation, which is the main assumption in this paper as mentioned in Introduction. We will have a few comments on this assumption in section 6 .

This proof also provides a holographic formula for calculating the capacity of entanglement $C$. Especially, the quantum fluctuation of the modular Hamiltonian with respect to the original state is given by

$$
\begin{aligned}
C(1) & =\left\langle H^{2}\right\rangle-\langle H\rangle^{2}, \\
& =\left.\int d^{d+1} X d^{d+1} X^{\prime} \frac{\delta G_{\mu \nu}(X)}{\delta n} \frac{\delta^{2} I}{\delta G_{\mu \nu}(X) \delta G_{\alpha \beta}\left(X^{\prime}\right)} \frac{\delta G_{\alpha \beta}\left(X^{\prime}\right)}{\delta n}\right|_{n=1} .
\end{aligned}
$$

To prove the third inequality $E \geq 0$ (2.20), we employ the expression (3.26) and it is enough to show $I_{\text {bulk }}\left[\hat{B}_{n}\right] \geq I_{\text {bulk }}\left[B_{1}\right]$ as $\hat{B}_{n}$ and $B_{1}$ obey the same boundary condition $\partial \hat{B}_{n}=\partial B_{1}=M_{1}$. It is so since the functional $I_{\text {bulk }}$ is supposed to have a minimum on the on-shell solution $B_{1}$, not the off-shell bulk $\hat{B}_{n}$, under the assumption that we can apply Gibbons-Hawking-Perry prescription so that the Euclidean gravity action $I_{\text {bulk }}$ is nonnegative definite. Instead, we can derive the third inequality $E \geq 0$ also from the second one $\tilde{S}_{n} \geq 0$ and the fourth one $C \geq 0$, in the same way as [18]. When $n \geq 1$, the free energy $F$ is non-negative because $\partial_{n} F=\tilde{S}_{n} / n^{2} \geq 0$ and $F(1)=0$, and so the energy $E=F+T \tilde{S}$ is also non-negative. The non-negativity of the capacity $d E / d T=C \geq 0$ means that the energy $E$ does not decrease with $T=1 / n$ and is still non-negative even when $n \leq 1$.

\subsection{Legendre transformed expression for capacity of entanglement}

We derive another expression of the entanglement heat capacity (4.9) using the graviton propagator, following [28] which calculates holographic entanglement entropies with probe branes inserted in the bulk.

\footnotetext{
${ }^{8}$ If we extend the domain of the integral from $\hat{B}_{n}$ to $B_{n}$ and use the action $I_{\text {bulk }}\left[B_{n}\right]=n I$, then the coefficient $n^{3}$ can be absorbed as

$$
C=\int_{B_{n}} d^{d+1} X d^{d+1} X^{\prime} \frac{\delta G_{\mu \nu}(X)}{\delta n} \frac{\delta^{2} I_{\mathrm{bulk}}\left[B_{n}\right]}{\delta G_{\mu \nu}(X) \delta G_{\alpha \beta}\left(X^{\prime}\right)} \frac{\delta G_{\alpha \beta}\left(X^{\prime}\right)}{\delta n} .
$$

This formula maybe applies to cases when the replica symmetry $\mathbb{Z}_{n}$ is spontaneously broken in the on-shell bulk $B_{n}$.
} 
We rewrite $\delta G_{\mu \nu} / \delta n$ appearing in (4.3), instead of $\delta \mathcal{A} / \delta G_{\mu \nu}$. By increasing the parameter $n$ slightly by $\delta n$, the energy-momentum tensor of the brane

$$
\bar{T}_{\mu \nu} \equiv \frac{\delta I}{\delta G^{\mu \nu}}=\frac{\sqrt{G}}{2} T_{\mu \nu}=T_{n} \frac{\delta \mathcal{A}}{\delta G^{\mu \nu}},
$$

changes slightly as

$$
\delta \bar{T}_{\mu \nu}=\frac{1}{4 G_{N}} \frac{\delta n}{n^{2}} \frac{\delta \mathcal{A}}{\delta G^{\mu \nu}} .
$$

Correspondingly the bulk metric $G_{\mu \nu}$ shifts by

$$
\begin{aligned}
\delta G_{\mu \nu}(X) & =8 \pi G_{N} \int d^{d+1} X^{\prime} G_{\mu \nu \alpha \beta}\left(X, X^{\prime}\right) 2 \delta \bar{T}^{\alpha \beta}\left(X^{\prime}\right), \\
& =-4 \pi \frac{\delta n}{n^{2}} \int d^{d+1} X^{\prime} G_{\mu \nu \alpha \beta}\left(X, X^{\prime}\right) \frac{\delta \mathcal{A}}{\delta G_{\alpha \beta}\left(X^{\prime}\right)} .
\end{aligned}
$$

Here $G_{\mu \nu \alpha \beta}$ is the Green's function of the linearized Einstein equation on the fixed background $G_{\mu \nu}$. Plugging it into (4.3), we obtain another expression of the entanglement heat capacity

$$
\begin{aligned}
C & =\frac{\pi}{n G_{N}} \int d^{d+1} X d^{d+1} X^{\prime} \frac{\delta \mathcal{A}}{\delta G_{\mu \nu}(X)} G_{\mu \nu \alpha \beta}\left(X, X^{\prime}\right) \frac{\delta A}{\delta G_{\alpha \beta}\left(X^{\prime}\right)}, \\
& =\left.\frac{1}{16 n G_{N}^{2}} \int d^{d+1} X d^{d+1} X^{\prime} \frac{\delta \mathcal{A}}{\delta G_{\mu \nu}(X)} \frac{\delta^{2} \log Z[\bar{T}]}{\delta \bar{T}_{\mu \nu}(X) \bar{T}_{\alpha \beta}\left(X^{\prime}\right)}\right|_{\bar{T}=0} \frac{\delta A}{\delta G_{\alpha \beta}\left(X^{\prime}\right)},
\end{aligned}
$$

where $Z[\bar{T}]$ is the partition function with a source $\bar{T}_{\mu \nu}$ inserted. $^{9}$ In this form, the nonnegativity of $C$ is equivalent to the concavity of $-\log Z\left[\bar{T}^{\prime}\right]$, which holds for $-\log Z[\bar{T}]$ is a Legendre transformation of the bulk action $-\log Z\left[G_{\mu \nu}(X)\right] \simeq I\left[G_{\mu \nu}(X)\right]$ as

$$
-\log Z[\bar{T}]=\min _{G_{\mu \nu}}\left(I\left[G_{\mu \nu}\right]-\int d^{d+1} X G_{\mu \nu}(X) \bar{T}^{\mu \nu}(X)\right),
$$

and in general the Legendre transformation $\mathcal{F}(J) \equiv \min _{M}[F(M)-J M]$ interchanges the convexity and the concavity, $\mathcal{F}^{\prime \prime}=-1 / F^{\prime \prime}$.

The explicit expression

$$
\frac{\delta \mathcal{A}}{\delta G^{\mu \nu}(X)}=-\frac{1}{2} \int d^{d-1} y \sqrt{g} g^{i j} \frac{\partial X^{\mu}}{\partial y^{i}} \frac{\partial X^{\nu}}{\partial y^{j}} \delta^{d+1}(X-X(y)),
$$

allows us to rewrite the formula with integrals on the brane

$$
C=\frac{\pi}{4 G_{N} n} \int d^{d-1} y d^{d-1} y^{\prime} \sqrt{g(y)} \sqrt{g\left(y^{\prime}\right)} \frac{\partial X^{\mu}}{\partial y^{i}} \frac{\partial X^{\nu}}{\partial y_{i}} G_{\mu \nu \alpha \beta}\left(X(y), X\left(y^{\prime}\right)\right) \frac{\partial X^{\alpha}}{\partial y^{\prime j}} \frac{\partial X^{\beta}}{\partial y_{j}^{\prime}} .
$$

\footnotetext{
${ }^{9}$ Here we assumed

$$
G_{\mu \nu \alpha \beta}\left(X, X^{\prime}\right)=\frac{1}{16 \pi G_{N}} \frac{\delta^{2} \log Z[\bar{T}]}{\delta \bar{T}_{\mu \nu}(X) \delta \bar{T}_{\alpha \beta}\left(X^{\prime}\right)},
$$

which could be shown by taking the variation of $\left\langle G_{\mu \nu}(X)\right\rangle_{\bar{T}}=\delta \log Z[\bar{T}] / \delta \bar{T}_{\mu \nu}(X)$, as

$$
\delta\left\langle G_{\mu \nu}(X)\right\rangle_{\bar{T}}=\int d^{d+1} X^{\prime} \frac{\delta^{2} \log Z[\bar{T}]}{\delta \bar{T}_{\mu \nu}(X) \delta \bar{T}_{\alpha \beta}\left(X^{\prime}\right)} \delta \bar{T}_{\alpha \beta}\left(X^{\prime}\right) .
$$

The normalization is determined by the definition of the graviton propagator (4.13)
} 
This representation is a consequence of the Legendre transformation between the response $G_{\mu \nu}$ and the source $\bar{T}^{\mu \nu}$. In fact, for a free energy $F\left(M_{i}\right)$ with general responses $M_{i}$ such as magnetization or chemical potential, the dual free energy $\mathcal{F}\left(J^{i}\right)$

$$
\mathcal{F}\left(J^{i}\right)=\min _{M_{i}}\left[F\left(M_{i}\right)-J^{i} M_{i}\right]
$$

with $J^{i}$ the dual sources such as magnetic field or charge, satisfies

$$
\delta M_{i} \frac{\partial^{2} F}{\partial M_{i} \partial M_{j}} \delta M_{j}=\delta J^{i} \delta M_{i}=-\delta J^{i} \frac{\partial^{2} \mathcal{F}}{\partial J^{i} \partial J^{j}} \delta J^{j},
$$

as $\delta F=J^{i} \delta M_{i}$ and $\delta \mathcal{F}=-M_{i} \delta J^{i}$. The Legendre transformation interchanges the convexity and the concavity.

\section{Calculations of the capacity of entanglement}

Our holographic proof of the inequalities for the Rényi entropy highlights a role of the stability in the bulk as a unitarity of the dual field theory. The discussion was illuminating for the formal proof, but less concrete so far. In this section, we switch gears and move onto tangible examples of the capacity of entanglement in various systems.

\subsection{Conformal field theory}

In two-dimensional CFT with central charge $c$ the Rényi entropies for an interval of length $L$ are well-known $[25,29]$

$$
S_{n}=\frac{c}{6}\left(1+\frac{1}{n}\right) \log (L / \epsilon),
$$

with the UV cutoff $\epsilon$. It yields the capacity of entanglement straightforwardly

$$
C(n)=\frac{c}{3 n} \log (L / \epsilon) .
$$

As it shows, the capacity is always positive in accord with the inequality (2.21) as the length $L$ cannot be smaller than the UV cutoff $\epsilon$.

It is challenging to obtain the capacity $C(n)$ for general $n$ in higher dimensional CFT, while one can calculate $C(n)$ of a sphere in the limit $n \rightarrow 1$. This is because $C(1)=$ $-\left.\partial_{n} \tilde{S}_{n}\right|_{n=1}$ is identical to the derivative of the Rényi entropy $C(1)=-\left.2 \partial_{n} S_{n}\right|_{n=1}$, whose calculations were already carried out for a sphere in CFT in [30]. In this case, the capacity becomes

$$
C(1)=\operatorname{Vol}\left(\mathbb{H}^{d-1}\right) \frac{2 \pi^{d / 2+1}(d-1) \Gamma(d / 2)}{\Gamma(d+2)} C_{T} .
$$

This is proportional to the coefficient $C_{T}$ of the correlation function of the energymomentum tensor [31]

$$
\left\langle T_{a b}(x) T_{c d}(0)\right\rangle=C_{T} \frac{I_{a b, c d}(x)}{x^{2 d}}
$$


where $I_{a b, c d}(x)$ is a function given by

$$
\begin{aligned}
I_{a b, c d}(x) & =\frac{1}{2}\left(I_{a c}(x) I_{b d}(x)+I_{a d}(x) I_{b c}(x)\right)-\frac{1}{d} \delta_{a b} \delta_{c d}, \\
I_{a b}(x) & =\delta_{a b}-2 \frac{x_{a} x_{b}}{x^{2}} .
\end{aligned}
$$

The positivity of $C(1)$ manifests itself in the form (5.3) as the volume of the hyperbolic space is positively divergent. In practice, it is convenient to introduce the regularized volume $^{10}$

$$
\operatorname{Vol}\left(\mathbb{H}^{d-1}\right)=\pi^{d / 2-1} \Gamma\left(1-\frac{d}{2}\right)
$$

to read off the so-called universal part of the Rényi entropies. This operation corresponds to adding local counter terms with respect to the background metric to render the partition function finite. It works well for any $d$ except even integers as the poles structure of the gamma function shows in (5.6). This signals the Weyl anomaly that cannot be removed by local counter terms. In even $d$ dimensions, one has to replace the formula $(5.6)$ with $[18,23]$

$$
\operatorname{Vol}\left(\mathbb{H}^{d-1}\right)=\frac{2(-\pi)^{d / 2-1}}{\Gamma(d / 2)} \log (R / \epsilon), \quad(d: \text { even }),
$$

by introducing the UV cutoff $\epsilon$ and the radius $R$ of the hyperbolic space. When applied to the entropy of an interval of width $L$ in $d=2$, the radius of the hyperbolic space $R$ should be identified with the width $L / 2$ in the regularized volume (5.7) and we are able to recover the $\mathrm{CFT}_{2}$ result (5.2) upon the relation $C_{T}=c /\left(2 \pi^{2}\right)$.

\subsection{Free fields}

The capacity of entanglement is less tractable to calculate for interacting QFTs as the modular Hamiltonian is non-local in general. For free field theories, things are much simpler and one is able to compute the Rényi entropies using the partition function on $\mathbb{S}^{1} \times \mathbb{H}^{d-1}$ which is conformally equivalent to the replica space of a spherical entangling surface $[23,32,33]$ (see also [34-36]).

Firstly we consider a conformally coupled real massless scalar field. With the help of the map to $\mathbb{S}^{1} \times \mathbb{H}^{d-1}$, the partition function on the $n$-fold replica manifold of a spherical entangling surface becomes [33]

$$
\log Z_{s}(n)=-\int_{0}^{\infty} d \lambda \mu_{s}(\lambda)\left[\log \left(1-e^{-2 \pi n \sqrt{\lambda}}\right)+\pi n \sqrt{\lambda}\right]
$$

where $\mu_{s}(\lambda)$ is the Plancherel measure of the scalar field on $\mathbb{H}^{d-1}[37,38]$

$$
\mu_{s}(\lambda)=\frac{\operatorname{Vol}\left(\mathbb{H}^{d-1}\right)}{2^{d-1} \pi^{\frac{d+1}{2}} \Gamma\left(\frac{d-1}{2}\right)} \sinh (\pi \sqrt{\lambda})\left|\Gamma\left(\frac{d}{2}-1+i \sqrt{\lambda}\right)\right|^{2} .
$$

\footnotetext{
${ }^{10}$ To derive (5.6), one can either put a cutoff near the infinity of the hyperbolic space, or use a dimensional regularization. In the former case, one ignores the power-law divergences for the cutoff to extract the universal part, while in the latter case one analytically continues the dimension $d$ from the range $1<d<2$ to an arbitrary value.
} 
Together with (2.18), it leads to the capacity of entanglement

$$
C_{s}(n)=\pi^{2} n^{2} \int_{0}^{\infty} d \lambda \mu_{s}(\lambda) \lambda \operatorname{csch}^{2}(\pi n \sqrt{\lambda}) .
$$

Turning into a massless Dirac fermion, the partition function is written as

$$
\log Z_{f}(n)=\int_{0}^{\infty} d \lambda \mu_{f}(\lambda)\left[\log \left(1+e^{-2 \pi n \lambda}\right)+\pi n \lambda\right]
$$

where the Plancherel measure of the spinor on $\mathbb{H}^{d-1}$ is [38]

$$
\mu_{f}(\lambda)=\frac{g(d) \operatorname{Vol}\left(\mathbb{H}^{d-1}\right)}{2^{d-2} \pi^{\frac{d+1}{2}} \Gamma\left(\frac{d-1}{2}\right)} \cosh (\pi \lambda)\left|\Gamma\left(\frac{d-1}{2}+i \lambda\right)\right|^{2}
$$

and $g(d) \equiv 2^{[d / 2]}$ is the dimension of Dirac spinors in $d$ dimensions. The capacity takes a similar form to the scalar field:

$$
C_{f}(n)=\pi^{2} n^{2} \int_{0}^{\infty} d \lambda \mu_{f}(\lambda) \lambda^{2} \operatorname{sech}^{2}(\pi n \lambda) .
$$

Both (5.10) and (5.13) are manifestly positive in their forms.

In two dimensions $d=2$, these capacities reproduces the $\mathrm{CFT}_{2}$ result (5.2) with $c=1$. They are also consistent with the general formula (5.3) of $C(1)$ for CFT where the free fields have the following values of $C_{T}[31]$

$$
\left(C_{T}\right)_{\text {scalar }}=\frac{d \Gamma(d / 2)^{2}}{4 \pi^{d}(d-1)}, \quad\left(C_{T}\right)_{\text {fermion }}=\frac{g(d) d \Gamma(d / 2)^{2}}{8 \pi^{d}}
$$

For massive cases and for a region $A$ other than a ball, it is hard to obtain capacities analytically, but we can resort to lattice discretization to calculate them numerically. The partition functions $\operatorname{Tr}\left[\rho_{A}^{n}\right]$ are expressed by correlation functions of discretized fields located in the region $A$ as follows [39-41]. For free scalars $\phi_{i}$ and its conjugates $\pi_{i}$ with correlation functions $X_{i j}=\left\langle\phi_{i} \phi_{j}\right\rangle$ and $P_{i j}=\left\langle\pi_{i} \pi_{j}\right\rangle$, the partition function is given by

$$
\log \operatorname{Tr}\left[\rho_{A}^{n}\right]=-\operatorname{Tr}\left[\log \left(\left(D_{s}+1 / 2\right)^{n}-\left(D_{s}-1 / 2\right)^{n}\right)\right]=-\sum_{a}\left(\log \left(e^{n \epsilon_{a}}-1\right)-n \log \left(e^{\epsilon_{a}}-1\right)\right),
$$

where we set the eigenvalues of $D_{s}=\sqrt{X P}(\geq 1 / 2)$ as $\operatorname{coth}\left(\epsilon_{a} / 2\right) / 2$. The indices $i, j$ run only the ones corresponding to the sites inside the region $A$. This yields a manifestly non-negative capacity

$$
C_{s}(n)=n^{2} \operatorname{Tr}\left[\frac{\left(D_{s}+1 / 2\right)^{n}\left(D_{s}-1 / 2\right)^{n}}{\left(\left(D_{s}+1 / 2\right)^{n}-\left(D_{s}-1 / 2\right)^{n}\right)^{2}}\left(\log \frac{D_{s}+1 / 2}{D_{s}-1 / 2}\right)^{2}\right]=\frac{n^{2}}{4} \sum_{a} \epsilon_{a}^{2} \operatorname{csch}^{2}\left(n \epsilon_{a} / 2\right) .
$$

The calculation for free fermions $\psi_{i}$ is similar [24]. The partition function given by

$$
\log \operatorname{Tr}\left[\rho_{A}^{n}\right]=\operatorname{Tr}\left[\log \left(\left(1-D_{f}\right)^{n}+D_{f}^{n}\right)\right]=\sum_{a}\left(\log \left(e^{n \epsilon_{a}}+1\right)-n \log \left(e^{\epsilon_{a}}+1\right)\right),
$$


yields a manifestly non-negative capacity

$$
C_{f}(n)=n^{2} \operatorname{Tr}\left[\frac{D_{f}^{n}\left(1-D_{f}\right)^{n}}{\left(D_{f}^{n}+\left(1-D_{f}\right)^{n}\right)^{2}}\left(\log \frac{D_{f}}{1-D_{f}}\right)^{2}\right]=\frac{n^{2}}{4} \sum_{a} \epsilon_{a}^{2} \operatorname{sech}^{2}\left(n \epsilon_{a} / 2\right),
$$

where the eigenvalues of the matrix $\left(D_{f}\right)_{i j}=\left\langle\psi_{i} \psi_{j}^{\dagger}\right\rangle$ are $1 /\left(1-e^{\epsilon_{a}}\right)$.

\subsection{Gravity duals}

The Rényi entropies of a spherical entangling surface are calculated through the holography using the AdS topological black hole [18]. The metric for the bulk per replica $\hat{B}_{n}$ is known to be

$$
d s_{d+1}^{2}=\frac{d r^{2}}{f_{n}(r)}+f_{n}(r) d \tau^{2}+r^{2}\left(d u^{2}+\sinh ^{2} u d \Omega_{d-2}^{2}\right)
$$

with a function

$$
f_{n}(r)=r^{2}-1-\frac{r_{n}^{d}-r_{n}^{d-2}}{r^{d-2}} .
$$

The Euclidean time direction $\tau$ has the period $\tau \sim \tau+2 \pi$ so that this metric reduces to the non-singular flat space $d \tau^{2}+d u^{2}+\sinh ^{2} u d \Omega_{d-2}^{2} \sim \sum_{i=1}^{d} d x_{i}^{2}$ at the conformal boundary $r \rightarrow$ $\infty$. This geometry has a conical singularity $C_{A}^{(n)}$ at the horizon $r=r_{n}\left(f_{n}\left(r_{n}\right)=0\right)$, where the Euclidean time $\tau$ circle shrinks to a point. The horizon radius $r_{n}$ is determined by $n$ as

$$
\begin{aligned}
\frac{2 \pi}{n}=\frac{f_{n}^{\prime}\left(r_{h}\right)}{2} 2 \pi & \Leftrightarrow \quad n=\frac{2}{f_{n}^{\prime}\left(r_{h}\right)}=\frac{2}{d r_{n}-(d-2) r_{n}^{-1}} \\
& \Leftrightarrow \quad r_{n}=\frac{1+\sqrt{1+n^{2} d(d-2)}}{n d},
\end{aligned}
$$

such that the correct conical singularity $\tau \sim \tau+2 \pi / n$ is reproduced. $r_{n}$ is monotonically decreasing with $n$ and satisfies $r_{n} \geq \lim _{n \rightarrow \infty} r_{n}=\sqrt{(d-2) / d}$.

The cosmic brane is located on the horizon and the improved Rényi entropy is nothing but the black hole entropy

$$
\tilde{S}_{n}=r_{n}^{d-1} \frac{\operatorname{Vol}\left(\mathbb{H}^{d-1}\right)}{4 G_{N}} .
$$

Integration by $n$ gives the free energy $F(n)$

$$
F(n)=\int_{1}^{n} d n^{\prime} \frac{\tilde{S}_{n^{\prime}}}{n^{\prime 2}}=\frac{\operatorname{Vol}\left(\mathbb{H}^{d-1}\right)}{4 G_{N}} \frac{2-r_{n}^{d}-r_{n}^{d-2}}{2}
$$

where we used the relation $\left(d+(d-2) / r_{n}^{2}\right) \partial_{n} r_{n}=-2 / n^{2}$ followed from the expression (5.21). This means that the Rényi entropy $S_{n}=n F /(n-1)$ is

$$
S_{n}=\frac{n}{n-1} \frac{\operatorname{Vol}\left(\mathbb{H}^{d-1}\right)}{4 G_{N}} \frac{2-r_{n}^{d}-r_{n}^{d-2}}{2}
$$

which is non-negative for any $n$ and $d$ as $r_{n}>1$ for $n<1$ and $r_{n}<1$ for $n>1$. We can also check that the first inequality (1.5) holds or equivalently $S\left[\rho_{n} \mid \rho\right]=-(n-1)^{2} \partial_{n} S_{n}=1+(d-$ 
1) $\left(r_{n}^{d}-r_{n}^{d-2}\right) / 2-r_{n}^{d-1} \geq 0$. The total energy (3.26) and the capacity of entanglement (4.1) given by

$$
\begin{aligned}
& E=\frac{\operatorname{Vol}\left(\mathbb{H}^{d-1}\right)}{4 G_{N}} \frac{2+(d-1)\left(r_{n}^{d}-r_{n}^{d-2}\right)}{2}, \\
& C=\frac{\operatorname{Vol}\left(\mathbb{H}^{d-1}\right)}{4 G_{N}}(d-1) r_{n}^{d-1} \frac{d r_{n}^{2}-(d-2)}{d r_{n}^{2}+(d-2)},
\end{aligned}
$$

are also non-negative for any $n$ and $d$ as $r_{n}>1$ for $n<1$ and $r_{n} \geq \sqrt{(d-2) / d}$ for $n>1$.

A more direct way to get $C$ without knowing $\tilde{S}_{n}$ is to use the formula

$$
C=-\frac{n}{8 G_{N}} \int_{C_{A}^{(n)}} d^{d-1} y \sqrt{g} g^{i j} \frac{\partial X^{\mu}}{\partial y^{i}} \frac{\partial X^{\nu}}{\partial y^{j}} \frac{\delta G_{\mu \nu}(X(y))}{\delta n}
$$

which is equivalent to the previous ones (4.3) and (4.19). ${ }^{11}$ When applying (5.28) to the background (5.19), we take the embedding $X^{\mu}(y)$ of the surface as $\left(X^{r}, X^{\tau}, X^{i}\right)=\left(1,0, y^{i}\right)$, where $y^{i}$ are the coordinates of $\mathbb{H}^{d-1}$. For $\mathbb{H}^{d-1}$ is maximally symmetric, the integration just gives its volume and the formula reads

$$
C=-\left.\frac{n \operatorname{Vol}\left(\mathbb{H}^{d-1}\right)}{8 G_{N}} r_{n}^{d-3} \frac{\delta G_{u u}}{\delta n}\right|_{C_{A}^{(n)}} .
$$

Reassuringly it agrees with (5.27) as $\delta G_{u u} / \delta n=\delta r_{n}^{2} / \delta n=2 r_{n} \partial_{n} r_{n}$.

When $n=1$, the holographic capacity of entanglement takes a particularly simple form

$$
C(1)=\frac{\operatorname{Vol}\left(\mathbb{H}^{d-1}\right)}{4 G_{N}} .
$$

It takes exactly the same form as the field theory calculation (5.3) because the holographic system has [42]

$$
C_{T}=\frac{1}{8 \pi G_{N}} \frac{d+1}{d-1} \frac{\Gamma(d+1)}{\pi^{d / 2} \Gamma(d / 2)} .
$$

One more example we are going to show is the system with two balls $A_{1}$ and $A_{2}$ of radii $R_{1}$ and $R_{2}$ separated enough (see figure 1 ). The Rényi entropy of the two balls for an arbitrary $n$ is beyond our scope, but a perturbative calculation is feasible in the leading linear order of $\delta n \equiv n-1$. Indeed, an analog of the mutual information $I^{(n)}\left(A_{1}, A_{2}\right) \equiv$ $S_{n}\left(A_{1}\right)+S_{n}\left(A_{2}\right)-S_{n}\left(A_{1} \cup A_{2}\right)$ has been evaluated holographically by [14] for $n$ close to 1 . We will benefit from the result to get the capacity of entanglement $C_{A_{1} \cup A_{2}}$ for the union of the two balls $A_{1}$ and $A_{2}$ in this parameter region.

The positions of the balls are parametrized by the cross ratio $0 \leq x \equiv \frac{\left(x_{1}-x_{2}\right)\left(x_{3}-x_{4}\right)}{\left(x_{1}-x_{3}\right)\left(x_{2}-x_{4}\right)} \leq$ 1. $x_{i}$ are the coordinates of the points where the line connecting the two centers intersects the balls, $x_{1,2}$ for $A_{1}$ and $x_{3,4}$ for $A_{2}$ (figure 1). There are two phases depending on the topology of the minimal surfaces in the bulk, and there is a critical point $x=x_{c}$ below

\footnotetext{
${ }^{11}$ Even when the graviton propagator $G_{\mu \nu \alpha \beta}\left(X, X^{\prime}\right)$ is known, the expression (4.19) is too difficult to evaluate in general and it suffers from a subtle contribution from the asymptotic boundary. We will comment on this difficulty in appendix A.
} 


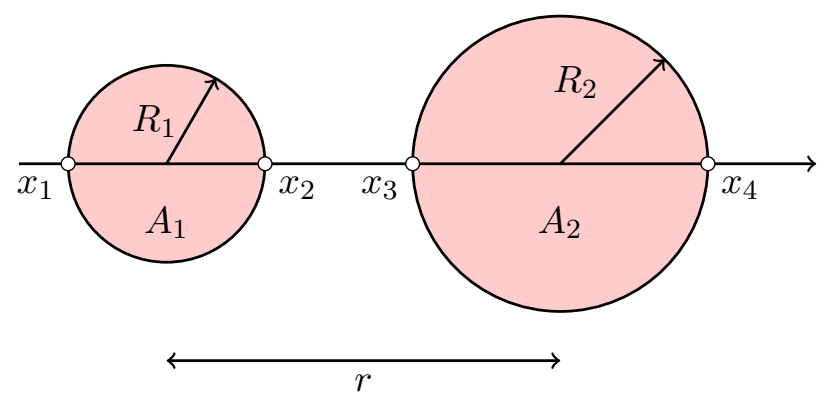

Figure 1. The entangling region (shown in red) consists of two balls $A_{1}$ and $A_{2}$ of radii $R_{1}$ and $R_{2}$, respectively. The four coordinates $x_{i}$ are defined on the line connecting the centers of the balls. In a conformal field theory, the configuration of the balls is uniquely specified by the cross ratio $x \equiv\left(x_{1}-x_{2}\right)\left(x_{3}-x_{4}\right) /\left(x_{1}-x_{3}\right)\left(x_{2}-x_{4}\right)=4 R_{1} R_{2} /\left(r^{2}-\left(R_{1}-R_{2}\right)^{2}\right)$, where $r$ is the distance between the two centers.

which a disconnected surface is favored, otherwise a connected one is realized [17]. The calculation of $I^{(n)}\left(A_{1}, A_{2}\right)$ performed by [14] is in the disconnected phase $\left(x \leq x_{c}\right)$ with the balls separated enough. To convert the result into the capacity $C(1)$, we apply a derivative $-\left.2 \partial_{n}\right|_{n=1}$ on $S_{n}\left(A_{1} \cup A_{2}\right)=S_{n}\left(A_{1}\right)+S_{n}\left(A_{2}\right)-I^{(n)}\left(A_{1}, A_{2}\right)$ to get

$$
\begin{aligned}
& C_{A_{1} \cup A_{2}}(1)=C_{A_{1}}(1)+C_{A_{2}}(1)+\left.2 \partial_{n} I^{(n)}\left(A_{1}, A_{2}\right)\right|_{n=1} \\
& =\frac{\operatorname{Vol}\left(\mathbb{H}^{d-1}\right)_{R_{1}}+\operatorname{Vol}\left(\mathbb{H}^{d-1}\right)_{R_{2}}}{4 G_{N}}+\frac{2^{4-d} \pi^{d+1} C_{T}}{d\left(d^{2}-1\right) \Gamma((d-1) / 2)^{2}} \frac{2-x}{x} B\left(\left(\frac{x}{2-x}\right)^{2} ; \frac{d+1}{2} ; \frac{2-d}{2}\right),
\end{aligned}
$$

where $\operatorname{Vol}\left(\mathbb{H}^{d-1}\right)_{R}$ is the regularized volume of $\mathbb{H}^{d-1}$ of radius $R$ given by (5.6) and (5.7).

\subsection{Large and small $n$ limits}

Before closing this section, we examine the large and small $n$ behaviours of the capacity $C(n)$ for a spherical entangling region in the systems we have studied. In the thermodynamic interpretation, we regard these as the low and high temperature limits for the temperature $T=1 / n$.

In the low temperature limit $n \rightarrow \infty$, the capacities of conformal theories go to zero as

$$
\begin{aligned}
C_{s}(n) & \sim \operatorname{Vol}\left(\mathbb{H}^{d-1}\right) \frac{\Gamma\left(\frac{d}{2}-1\right)^{2}}{15 \cdot 2^{d-1} \pi^{\frac{d-3}{2}} \Gamma\left(\frac{d-1}{2}\right)} \frac{1}{n^{3}} \quad(d \neq 2), \\
C_{f}(n) & \sim \operatorname{Vol}\left(\mathbb{H}^{d-1}\right) \frac{g(d)) \Gamma\left(\frac{d-1}{2}\right)}{3 \cdot 2^{d} \pi^{\frac{d-1}{2}}} \frac{1}{n}, \\
C_{\mathrm{AdS}}(n) & \sim \frac{\operatorname{Vol}\left(\mathbb{H}^{d-1}\right)}{4 G_{N}} \frac{(d-1)(d-2)^{d / 2-1}}{d^{d / 2}} \frac{1}{n},
\end{aligned}
$$

for the massless scalar, massless fermion and CFT dual to the AdS spacetime, respectively. They are proportional to a power of the temperature $T=1 / n$, indicating a gapless excitation for the modular Hamiltonian. In $d=2$, the scalar capacity also becomes proportional to $1 / n$ as $C_{s}(n)=(c / 3 n) \log (L / \epsilon)$. 
On the other hand, in the high temperature limit $n \rightarrow 0$, they obey the StefanBoltzmann's law $C(T) \propto T^{d-1}$ for thermal massless gases

$$
\begin{aligned}
C_{s}(n) & \sim \operatorname{Vol}\left(\mathbb{H}^{d-1}\right) \frac{(d-1) \Gamma(d / 2+1) \zeta(d)}{2^{d-2} \pi^{\frac{3}{2} d-1}} \frac{1}{n^{d-1}}, \\
C_{f}(n) & \sim \operatorname{Vol}\left(\mathbb{H}^{d-1}\right) \frac{(d-1)\left(2^{d-1}-1\right) \Gamma(d / 2+1) \zeta(d) g(d)}{2^{2 d-3} \pi^{\frac{3}{2} d-1}} \frac{1}{n^{d-1}}, \\
C_{\mathrm{AdS}}(n) & \sim \frac{\operatorname{Vol}\left(\mathbb{H}^{d-1}\right)}{4 G_{N}}(d-1)\left(\frac{2}{n d}\right)^{d-1} .
\end{aligned}
$$

To derive these results, we used asymptotic behavior of $\mu(\lambda)$

$$
\mu_{s}(\lambda) \sim \frac{\operatorname{Vol}\left(\mathbb{H}^{d-1}\right)}{2^{d-1} \pi^{\frac{d-1}{2}} \Gamma\left(\frac{d-1}{2}\right)} \lambda^{\frac{d-3}{2}}, \quad \mu_{f}(\lambda) \sim \frac{g(d) \operatorname{Vol}\left(\mathbb{H}^{d-1}\right)}{2^{d-2} \pi^{\frac{d-1}{2}} \Gamma\left(\frac{d-1}{2}\right)} \lambda^{d-2},
$$

in the limit $\lambda \rightarrow \infty$ and mathematical relations

$$
\begin{aligned}
\int_{0}^{\infty} d x x^{d} \operatorname{csch}^{2} x & =\frac{\Gamma(d+1) \zeta(d)}{2^{d-1}}, \\
\int_{0}^{\infty} d x x^{d} \operatorname{sech}^{2} x & =\frac{\left(2^{d-1}-1\right) \Gamma(d+1) \zeta(d)}{4^{d-1}},
\end{aligned}
$$

and $\Gamma(d+1) / 2^{d}=\Gamma\left(\frac{d+1}{2}\right) \Gamma\left(\frac{d}{2}+1\right) / \sqrt{\pi}$.

\section{Discussion}

Our approach to the holographic Rényi entropy is advantageous for formal proofs and provides a clear-cut relation of the roles played by the unitarity in QFT and the stability of the gravity theory. Meanwhile, the holographic formula lacks a power of computability in a practical problems as we saw in section 5. The main difficulty originates from the procedure of finding the extremal surface of a cosmic brane in the backreacted geometry. One would be able to calculate the Rényi entropy perturbatively either in $n-1$ or in shape, otherwise it is generically unattainable in its nature. It is still algorithmically simple to implement in numerical calculation that would be worth more investigation.

We do not know any rigorous proof or plausible argument for the bulk stability against any perturbation that is essential in our holographic proof of the inequalities. To answer a question whether the bulk is stable or not requires the knowledge of quantum gravity which remains to be developed. It is one of the fundamental problems even in the perturbative Euclidean quantum gravity and providing the complete solution is far beyond the scope of this paper. We comment on possible attempts instead:

- The assumption we made for the bulk stability is a sufficient condition, but may not be a necessary condition, to prove the Rényi entropic inequalities in the holographic system. Namely the non-negativity of the heat capacity (4.9) could have followed from the condition for the Hessian matrix to be non-negative definite only in the subspace of the metric variation $\delta G_{\mu \nu} / \delta n$ induced by changing the replica parameter. 
Unfortunately we were not able to demonstrate the non-negativity of the Hessian in the subspace as the metric variation is only calculable in the neighbourhood of the cosmic brane.

- The perturbative Euclidean gravity is known to suffer from the bulk instability due to the Weyl mode. ${ }^{12}$ There are at least two directions known in literature to fix this problem: one (ad-hoc) attempt is Gibbons-Hawking-Perry prescription which claims to change the contour of integration for the Weyl mode, called conformal rotation, in the path integral formulation of the perturbative Euclidean gravity [21, 22, 43]. (See also $[44,45]$ for further discussions.) For locally Euclidean $\mathrm{AdS}_{3}$ spaces, this prescription gives the correct one-loop partition function of gravity expected from the $\mathrm{AdS}_{3} / \mathrm{CFT}_{2}$ correspondence [46], and it might well work for more general holographic theories at the one-loop level. The other is based on the canonical quantization of gravity to show the Hamiltonian is bounded from below, and then continues to Euclidean path integral with an appropriate choice of contour [47, 48]. The two approaches appear to be complimentary to each other, but a precise relation between them has not been completely explored.

As a future direction, it would also be intriguing to include quantum corrections to the holographic Rényi entropy [49]. Recent discussions [50, 51] argues a relation between the boundary modular Hamiltonian $H_{\text {bdy }}$ and the bulk one $H_{\text {bulk }}$

$$
H_{\text {bdy }}=\frac{\hat{A}}{4 G_{N}}+H_{\text {bulk }}+\hat{S}_{\text {Wald-like }}+O\left(G_{N}\right) .
$$

Here $\hat{A}$ is an operator in the bulk which is supposed to give the area of the Ryu-Takayanagi surface $\mathcal{S}$ when sandwiched by a state dual to a given state in the boundary field theory.

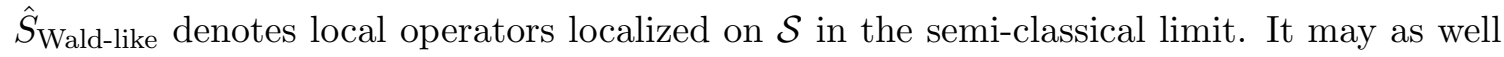
be applied to the calculation of the capacity (2.18) for $n=1$, leading to

$$
C(1)_{\mathrm{bdy}}=\frac{1}{16 G_{N}^{2}}\left(\left\langle\hat{A}^{2}\right\rangle-\langle\hat{A}\rangle^{2}\right)+\frac{1}{2 G_{N}}\left(\left\langle\hat{A} \tilde{H}_{\mathrm{bulk}}\right\rangle-\langle\hat{A}\rangle\left\langle\tilde{H}_{\mathrm{bulk}}\right\rangle\right)+O(1)
$$

where we introduced $\tilde{H}_{\text {bulk }} \equiv H_{\text {bulk }}+\hat{S}_{\text {Wald-like }}$ to simplify the notation. Surprisingly, the leading term is of order $1 / G_{N}^{2}$, which was not observed in the examples in section 5 . Thus we are lead to conclude that the area operator has to satisfy

$$
\alpha \equiv \frac{\left\langle\hat{A}^{2}\right\rangle-\langle\hat{A}\rangle^{2}}{8 G_{N}}=O\left(G_{N}^{0}\right) .
$$

We believe this is a defining property of the area operator that holds for any state in the semi-classical limit. A similar statement has been made in, e.g., [52] in the context of the linearity of the area operator recently. The order $1 / G_{N}$ term is likely to contribute to the capacity, and it indeed does so for the cases considered in section 5. We do not know how to estimate it in practice, but the non-negativity of the capacity yields a constraint

$$
\alpha+\left\langle\hat{A} \tilde{H}_{\text {bulk }}\right\rangle-\langle\hat{A}\rangle\left\langle\tilde{H}_{\text {bulk }}\right\rangle \geq 0+O\left(G_{N}\right) .
$$

\footnotetext{
${ }^{12}$ We thank M. Headrick for drawing our attention to this subtlety.
} 
Testing this inequality needs more detailed information on the area operator and the local operators on the Ryu-Takayanagi surface $\mathcal{S}$, which is far beyond the scope of the present work.

Another interesting direction is to generalize the holographic formula of the Rényi entropy to a time dependent background [53] and higher derivative gravities [54, 55]. It is not so obvious how a cosmic brane modifies the original proposals, but it is likely that the entropy is still given by variants of the area formula.

\section{Acknowledgments}

We are grateful to T. Kawano, Y. Nakagawa and Y. Sato for valuable discussions. We also thank the Yukawa Institute for Theoretical Physics for hospitality during the completion of the paper. The work of YN was supported by World Premier International Research Center Initiative (WPI), MEXT, Japan. The work of YN was also supported in part by JSPS Research Fellowship for Young Scientists. The work of TN was supported in part by JSPS Grant-in-Aid for Young Scientists (B) No. 15K17628.

\section{A On holographic calculation of $C(1)$ using graviton propagator}

In this appendix, we use the expression (4.19) including the graviton propagator to calculate $C(1)$ for a spherical entangling surface. First, we reproduce the formula

$$
C(n)=\frac{\pi}{4 G_{N} n} \int d^{d-1} y d^{d-1} y^{\prime} \sqrt{g(y)} \sqrt{g\left(y^{\prime}\right)} J\left(y, y^{\prime}\right),
$$

where

$$
J\left(y, y^{\prime}\right) \equiv \frac{\partial X^{\mu}}{\partial y^{i}} \frac{\partial X^{\nu}}{\partial y_{i}} G_{\mu \nu \alpha \beta}\left(X(y), X\left(y^{\prime}\right)\right) \frac{\partial X^{\alpha}}{\partial y^{\prime j}} \frac{\partial X^{\beta}}{\partial y_{j}^{\prime}} .
$$

There is a difficulty related to the boundary term in this formula as commented in [28] and pointing it out is the purpose of this appendix.

The graviton propagator $G_{\mu \nu \alpha \beta}$ is not known for the backreacted metric with general $n$, while the metric is just $A d S_{d+1}$ for $n=1$ whose graviton propagator $G_{\mu \nu \mu^{\prime} \nu^{\prime}}\left(X, X^{\prime}\right)$ can be represented as [56]

$$
G_{\mu \nu \mu^{\prime} \nu^{\prime}}\left(X, X^{\prime}\right)=\left(\partial_{\mu} \partial_{\mu^{\prime}} D \partial_{\nu} \partial_{\nu^{\prime}} D+(\mu \leftrightarrow \nu)\right) G(D)+G_{\mu \nu}(X) G_{\mu^{\prime} \nu^{\prime}}\left(X^{\prime}\right) H(D)+\cdots
$$

The $(\cdots)$ terms are gauge-dependent and do not matter when the bulk energy momentum tensor $T_{\mu \nu}$ vanishes at the boundary fast enough, but they would contribute in the current setup because the energy-momentum tensor of the brane does not decay at the boundary. The $(\cdots)$ term is too complicated to be taken into account, and we proceed without having them for a moment.

We are going to evaluate the $G(D)$ and $H(D)$ parts. $\partial_{\mu}=\partial / \partial X^{\mu}$ and $\partial_{\mu^{\prime}}=\partial / \partial X^{\prime \mu^{\prime}}$ are derivatives with respect to the bulk points $X$ and $X^{\prime}$. The two functions $G(D)$ and 
$H(D)$ are given by

$$
\begin{aligned}
& G(D)=\tilde{C}_{d}\left(\frac{2}{D}\right)^{d} F\left(d, \frac{d+1}{2} ; d+1 ;-\frac{2}{D}\right), \\
& H(D)=-\frac{2(D+1)^{2}}{d-1} G(D)+\frac{4(d-2)(D+1)}{(d-1)^{2}} \tilde{C}_{d}\left(\frac{2}{D}\right)^{d-1} F\left(d-1, \frac{d+1}{2} ; d+1 ;-\frac{2}{D}\right),
\end{aligned}
$$

with a constant

$$
\tilde{C}_{d}=\frac{\Gamma\left(\frac{d+1}{2}\right)}{(4 \pi)^{\frac{d+1}{2}} d}=\frac{1}{2^{d} d \operatorname{Vol}\left(\mathbb{S}^{d}\right)} .
$$

The function $D=D\left(X, X^{\prime}\right)$ is the invariant distance between the two points $X$ and $X^{\prime}$,

$$
D=\frac{1}{2}\left[-\left(X_{-1}^{\prime}-X_{-1}\right)^{2}+\left(X_{0}^{\prime}-X_{0}\right)^{2}+\left(X_{1}^{\prime}-X_{1}\right)^{2}+\cdots+\left(X_{d}^{\prime}-X_{d}\right)^{2}\right],
$$

in the Euclidean $A d S_{d+1}$ space realized as an embedding $-X_{-1}^{2}+X_{0}^{2}+X_{1}^{2}+\cdots+X_{d}^{2}=-1$ in $\mathbb{R}^{1, d+1}$, with the metric $d s^{2}=-d X_{-1}^{2}+d X_{0}^{2}+d X_{1}^{2}+\cdots+d X_{d}^{2}$. An expression of $D$ in the hyperbolic coordinate

$$
d s_{d+1}^{2}=\frac{d r^{2}}{r^{2}-1}+\left(r^{2}-1\right) d \tau^{2}+r^{2}\left(d u^{2}+\sinh ^{2} u d \Omega_{d-2}^{2}\right),
$$

follows from the coordinate transformation

$$
\begin{aligned}
X_{-1} & =r \cosh u, & X_{i} & =r \sinh u \Omega_{i-1} \quad(i=2, \ldots, d), \\
X_{0} & =\sqrt{r^{2}-1} \sin \tau, & X_{1} & =\sqrt{r^{2}-1} \cos \tau .
\end{aligned}
$$

The minimal surface is the horizon $r=1$ of the topological black hole at $\tau=0$, on which the invariant distance $D$ becomes

$$
D\left(u^{\prime}, \Omega_{i}^{\prime} ; u, \Omega_{i}\right)=\cosh u \cosh u^{\prime}-\sinh u \sinh u^{\prime} \sum_{i=1}^{d-1} \Omega_{i} \Omega_{i}^{\prime}-1 .
$$

The function $J$ is calculated as ${ }^{13,14}$

$$
J=2\left((D+1)^{2}+d-2\right) G(D)+(d-1)^{2} H(D)+\cdots,
$$

\footnotetext{
${ }^{13}$ To calculate $J=J(D)$, it is easier to work in Poincaré coordinate $d s_{d+1}^{2}=\left(d z^{2}+\sum_{i=0}^{d-1} d x_{i}^{2}\right) / z^{2}$ related by

$$
X_{-1}=\frac{z}{2}+\frac{1+\sum_{i=0}^{d-1} x_{i}^{2}}{2 z}, \quad X_{i}=\frac{x_{i}}{z} \quad(i=0, \ldots, d-1), \quad X_{d}=\frac{z}{2}+\frac{-1+\sum_{i=0}^{d-1} x_{i}^{2}}{2 z},
$$

because the minimal surface is mapped to just a plane $x^{0}=x^{1}=0$.

${ }^{14}$ The $(\cdots)$ term would be represented as

$$
\begin{aligned}
(\cdots)= & 2\left(d(D+1)^{2}-1\right) X(D)+2 D(D+1)(D+2) X^{\prime}(D) \\
& +2(d+1) D(D+1)(D+2) Y(D)+2 D^{2}(D+2)^{2} Y^{\prime}(D) \\
& +2(d-1)^{2}(D+1) Z(D)+2(d-1) D(D+2) Z^{\prime}(D),
\end{aligned}
$$

with functions $X, Y, Z$ given implicitly in [28].
} 
which is just a function of the invariant distance $D$. The symmetry of the hyperbolic space $\mathbb{H}^{d-1}$ allows us to move the two points to $\left(u^{\prime}, \Omega^{\prime}\right)=(0,0)$ and $(u, \Omega)=(u, 0)$, and factor out the integrals over $u^{\prime}, \Omega^{\prime}$ and $\Omega$ :

$$
\begin{aligned}
C(1) & =\frac{\pi}{4 G_{N}} \int d u d u^{\prime} d \Omega_{d-2} d \Omega_{d-2}^{\prime} \sinh ^{d-2} u \sinh ^{d-2} u^{\prime} J(D), \\
& =\frac{\operatorname{Vol}\left(\mathbb{H}^{d-1}\right)}{4 G_{N}} \pi \operatorname{Vol}\left(\mathbb{S}^{d-2}\right) \int d u \sinh ^{d-2} u J(D),
\end{aligned}
$$

where $D=D(u, 0 ; 0,0)=\cosh u-1$. The integration of $G(D)$ and $H(D)$ parts of $J(D)$ can be performed as

$$
C(1)=\frac{\operatorname{Vol}\left(\mathbb{H}^{d-1}\right)}{4 G_{N}}\left(\frac{d-2}{d}+\cdots\right) .
$$

Compared with the previous result (5.30), we speculate that the gauge-dependent part contributes $2 / d$. It would be desirable to include the gauge-dependent contribution in order to confirm our conjecture, but we leave it to future investigations.

\section{B Comments on the strong sub-additivity of Rényi entropies}

We have given a holographic proof of the Rényi entropic inequalities, but they are not related to the strong sub-additivity of entanglement entropy (1.2). In fact, the Rényi entropy $S_{n}$ is nether strong sub-additive nor sub-additive (1.3). The improved Rényi entropy $\tilde{S}_{n}(2.10)$ does not satisfy them too.

To achieve the sub-additivity and strong sub-additivity of the Rényi entropy, it would be helpful to review how these inequalities are related to information theoretic measures. In fact, these inequalities follow from properties of the relative entropy $S[\rho \mid \sigma] \equiv$ $\operatorname{Tr}[\rho(\log \rho-\log \sigma)]$. Relative entropy is non-negative $S[\rho \mid \sigma] \geq 0$, and equivalently the mutual information

$$
I(A, B)=S_{A}+S_{B}-S_{A B}=S\left[\rho_{A B} \mid \rho_{A} \otimes \rho_{B}\right] \geq 0
$$

is also non-negative. These are also equivalent to the sub-additivity of entanglement entropy. On the other hand, the strong sub-additivity of entanglement entropy is equivalent to the non-negativity of the conditional mutual information

$$
\begin{aligned}
I(A, B \mid C) & \equiv S_{A \mid C}+S_{B \mid C}-S_{A B \mid C}, \\
& =S_{A C}+S_{B C}-S_{A B C}-S_{C},
\end{aligned}
$$

where $S_{X \mid Y} \equiv S_{X Y}-S_{Y}$ is called the conditional entropy. Relative entropy decreases monotonically under partial trace, $S[\rho \mid \sigma] \geq S\left[\operatorname{Tr}_{B} \rho \mid \operatorname{Tr}_{B} \sigma\right]$, meaning that the conditional mutual information $I(A, B \mid C)$ is non-negative

$$
\begin{aligned}
I(A, B \mid C) & =S\left[\rho_{A B C} \mid \rho_{A} \otimes \rho_{B C}\right]-S\left[\rho_{A C} \mid \rho_{A} \otimes \rho_{C}\right] \\
& =S\left[\rho_{A B C} \mid \rho_{A} \otimes \rho_{B C}\right]-S\left[\operatorname{Tr}_{B}\left[\rho_{A B C}\right] \mid \operatorname{Tr}_{B}\left[\rho_{A} \otimes \rho_{B C}\right]\right] \\
& \geq 0
\end{aligned}
$$


Now one way to generalize these inequalities to the Rényi entropy is introducing the relative entropy or mutual information for the Rényi entropy. One promising proposal of the relative Rényi entropy is $[57,58]$

$$
S_{n}[\rho \mid \sigma] \equiv \frac{1}{n-1} \log \operatorname{Tr}\left[\left(\sigma^{\frac{1-n}{2 n}} \rho \sigma^{\frac{1-n}{2 n}}\right)^{n}\right],
$$

which reduces to the relative entropy $S[\rho \mid \sigma]$ in the limit $n \rightarrow 1$. This generalization of the relative entropy keeps the non-negativity $S_{n}[\rho \mid \sigma] \geq 0$ and monotonicity $S_{n}[\rho \mid \sigma] \geq$ $S_{n}\left[\operatorname{Tr}_{B} \rho \mid \operatorname{Tr}_{B} \sigma\right]$ under partial trace [59]. So we assert that the Rényi generalization of the sub-additivity would be

$$
I_{n}(A, B) \equiv S_{n}\left[\rho_{A B} \mid \rho_{A} \otimes \rho_{B}\right] \geq 0
$$

and the Rényi generalization of the strong sub-additivity would be

$$
S_{n}\left[\rho_{A B C} \mid \rho_{A} \otimes \rho_{B C}\right]-S_{n}\left[\rho_{A C} \mid \rho_{A} \otimes \rho_{C}\right] \geq 0 .
$$

For entanglement entropy with $n=1$, these inequalities admit a holographic interpretation. For the Rényi entropy for any $n$, however, it is not possible to express the relative Rényi entropy $S_{n}[\rho \mid \sigma]$ or Rényi mutual information $I_{n}$ as a linear combination of the Rényi entropies. So it is not clear how to interpret these Rényi-generalized inequalities holographically, even though we have the holographic Rényi entropy formula. The expression of the relative Rényi entropy (B.4) suggests that it can be calculated by the replica method [60] and it may have an interpretation and proof of these Rényi-generalized inequalities in a holographic system.

Open Access. This article is distributed under the terms of the Creative Commons Attribution License (CC-BY 4.0), which permits any use, distribution and reproduction in any medium, provided the original author(s) and source are credited.

\section{References}

[1] S. Ryu and T. Takayanagi, Holographic derivation of entanglement entropy from AdS/CFT, Phys. Rev. Lett. 96 (2006) 181602 [hep-th/0603001] [InSPIRE].

[2] L. Susskind, The world as a hologram, J. Math. Phys. 36 (1995) 6377 [hep-th/9409089] [INSPIRE].

[3] G. 't Hooft, Dimensional reduction in quantum gravity, gr-qc/9310026 [INSPIRE].

[4] T. Takayanagi, Entanglement entropy from a holographic viewpoint, Class. Quant. Grav. 29 (2012) 153001 [arXiv:1204.2450] [INSPIRE].

[5] D. Harlow, Jerusalem lectures on black holes and quantum information, Rev. Mod. Phys. 88 (2016) 15002 [arXiv:1409.1231] [InSPIRE].

[6] E.H. Lieb and M.B. Ruskai, Proof of the strong subadditivity of quantum-mechanical entropy, J. Math. Phys. 14 (1973) 1938 [inSPIRE].

[7] H. Casini and M. Huerta, A c-theorem for the entanglement entropy, J. Phys. A 40 (2007) 7031 [cond-mat/0610375] [INSPIRE]. 
[8] H. Casini and M. Huerta, On the RG running of the entanglement entropy of a circle, Phys. Rev. D 85 (2012) 125016 [arXiv: 1202.5650] [InSPIRE].

[9] M. Headrick and T. Takayanagi, A holographic proof of the strong subadditivity of entanglement entropy, Phys. Rev. D 76 (2007) 106013 [arXiv:0704.3719] [INSPIRE].

[10] A.C. Wall, Maximin surfaces and the strong subadditivity of the covariant holographic entanglement entropy, Class. Quant. Grav. 31 (2014) 225007 [arXiv:1211.3494] [inSPIRE].

[11] M. Headrick, General properties of holographic entanglement entropy, JHEP 03 (2014) 085 [arXiv: 1312.6717] [INSPIRE].

[12] P. Hayden, M. Headrick and A. Maloney, Holographic mutual information is monogamous, Phys. Rev. D 87 (2013) 046003 [arXiv:1107.2940] [inSPIRE].

[13] N. Bao, S. Nezami, H. Ooguri, B. Stoica, J. Sully and M. Walter, The holographic entropy cone, JHEP 09 (2015) 130 [arXiv: 1505.07839] [INSPIRE].

[14] X. Dong, The gravity dual of Rényi entropy, Nature Commun. 7 (2016) 12472 [arXiv: 1601.06788] [INSPIRE].

[15] A. Lewkowycz and J. Maldacena, Generalized gravitational entropy, JHEP 08 (2013) 090 [arXiv: 1304.4926] [INSPIRE].

[16] D.V. Fursaev, Proof of the holographic formula for entanglement entropy, JHEP 09 (2006) 018 [hep-th/0606184] [INSPIRE].

[17] M. Headrick, Entanglement Rényi entropies in holographic theories, Phys. Rev. D 82 (2010) 126010 [arXiv:1006.0047] [INSPIRE].

[18] L.-Y. Hung, R.C. Myers, M. Smolkin and A. Yale, Holographic calculations of Rényi entropy, JHEP 12 (2011) 047 [arXiv:1110.1084] [INSPIRE].

[19] K. Życzkowski, Rényi extrapolation of Shannon entropy, Open Syst. Inf. Dyn. 10 (2003) 297 [quant-ph/0305062].

[20] C. Beck and F. Schögl, Thermodynamics of chaotic systems, Cambridge University Press, Cambridge U.K. (1993).

[21] G.W. Gibbons, S.W. Hawking and M.J. Perry, Path integrals and the indefiniteness of the gravitational action, Nucl. Phys. B 138 (1978) 141 [inSPIRE].

[22] S.M. Christensen and M.J. Duff, Quantizing gravity with a cosmological constant, Nucl. Phys. B 170 (1980) 480 [inSPIRE].

[23] H. Casini, M. Huerta and R.C. Myers, Towards a derivation of holographic entanglement entropy, JHEP 05 (2011) 036 [arXiv:1102.0440] [INSPIRE].

[24] H. Yao and X.-L. Qi, Entanglement entropy and entanglement spectrum of the Kitaev model, Phys. Rev. Lett. 105 (2010) 080501 [arXiv:1001.1165] [INSPIRE].

[25] P. Calabrese and J.L. Cardy, Entanglement entropy and quantum field theory, J. Stat. Mech. 06 (2004) P06002 [hep-th/0405152] [InSPIRE].

[26] J. Camps and W.R. Kelly, Generalized gravitational entropy without replica symmetry, JHEP 03 (2015) 061 [arXiv: 1412.4093] [INSPIRE].

[27] D.V. Fursaev and S.N. Solodukhin, On the description of the Riemannian geometry in the presence of conical defects, Phys. Rev. D 52 (1995) 2133 [hep-th/9501127] [INSPIRE]. 
[28] H.-C. Chang and A. Karch, Entanglement entropy for probe branes, JHEP 01 (2014) 180 [arXiv:1307.5325] [INSPIRE].

[29] C. Holzhey, F. Larsen and F. Wilczek, Geometric and renormalized entropy in conformal field theory, Nucl. Phys. B 424 (1994) 443 [hep-th/9403108] [INSPIRE].

[30] E. Perlmutter, A universal feature of CFT Rényi entropy, JHEP 03 (2014) 117 [arXiv: 1308.1083] [INSPIRE].

[31] H. Osborn and A.C. Petkou, Implications of conformal invariance in field theories for general dimensions, Annals Phys. 231 (1994) 311 [hep-th/9307010] [INSPIRE].

[32] H. Casini and M. Huerta, Entanglement entropy for the $N$-sphere, Phys. Lett. B 694 (2011) 167 [arXiv:1007.1813] [INSPIRE].

[33] I.R. Klebanov, S.S. Pufu, S. Sachdev and B.R. Safdi, Rényi entropies for free field theories, JHEP 04 (2012) 074 [arXiv:1111.6290] [InSPIRE].

[34] J.S. Dowker, Entanglement entropy for even spheres, arXiv:1009.3854 [INSPIRE].

[35] J.S. Dowker, Entanglement entropy for odd spheres, arXiv: 1012.1548 [INSPIRE].

[36] S.N. Solodukhin, Entanglement entropy of round spheres, Phys. Lett. B 693 (2010) 605 [arXiv: 1008.4314] [INSPIRE].

[37] R. Camporesi, Harmonic analysis and propagators on homogeneous spaces, Phys. Rept. 196 (1990) 1 [INSPIRE].

[38] A.A. Bytsenko, G. Cognola, L. Vanzo and S. Zerbini, Quantum fields and extended objects in space-times with constant curvature spatial section, Phys. Rept. 266 (1996) 1 [hep-th/9505061] [INSPIRE].

[39] M. Srednicki, Entropy and area, Phys. Rev. Lett. 71 (1993) 666 [hep-th/9303048] [INSPIRE].

[40] I. Peschel, Calculation of reduced density matrices from correlation functions, J. Phys. A 36 (2003) L205 [cond-mat/0212631].

[41] H. Casini and M. Huerta, Entanglement entropy in free quantum field theory, J. Phys. A 42 (2009) 504007 [arXiv:0905.2562] [InSPIRE].

[42] A. Buchel, J. Escobedo, R.C. Myers, M.F. Paulos, A. Sinha and M. Smolkin, Holographic GB gravity in arbitrary dimensions, JHEP 03 (2010) 111 [arXiv:0911.4257] [INSPIRE].

[43] S.W. Hawking, The path integral approach to quantum gravity, in General relativity: an Einstein centenary survey, (1980), pg. 746 [INSPIRE].

[44] P.O. Mazur and E. Mottola, The gravitational measure, solution of the conformal factor problem and stability of the ground state of quantum gravity, Nucl. Phys. B 341 (1990) 187 [INSPIRE].

[45] E. Mottola, Functional integration over geometries, J. Math. Phys. 36 (1995) 2470 [hep-th/9502109] [INSPIRE].

[46] S. Giombi, A. Maloney and X. Yin, One-loop partition functions of $3 D$ gravity, JHEP 08 (2008) 007 [arXiv:0804.1773] [INSPIRE].

[47] H. Arisue, T. Fujiwara, M. Kato and K. Ogawa, Path integral and operator formalism in quantum gravity, Phys. Rev. D 35 (1987) 2309 [InSPIRE].

[48] K. Schleich, Conformal rotation in perturbative gravity, Phys. Rev. D 36 (1987) 2342 [INSPIRE]. 
[49] T. Faulkner, A. Lewkowycz and J. Maldacena, Quantum corrections to holographic entanglement entropy, JHEP 11 (2013) 074 [arXiv: 1307.2892] [INSPIRE].

[50] D.L. Jafferis and S.J. Suh, The gravity duals of modular Hamiltonians, JHEP 09 (2016) 068 [arXiv: 1412.8465] [INSPIRE].

[51] D.L. Jafferis, A. Lewkowycz, J. Maldacena and S.J. Suh, Relative entropy equals bulk relative entropy, JHEP 06 (2016) 004 [arXiv: 1512.06431] [INSPIRE].

[52] A. Almheiri, X. Dong and B. Swingle, Linearity of holographic entanglement entropy, arXiv: 1606.04537 [INSPIRE].

[53] V.E. Hubeny, M. Rangamani and T. Takayanagi, A covariant holographic entanglement entropy proposal, JHEP 07 (2007) 062 [arXiv:0705.0016] [INSPIRE].

[54] X. Dong, Holographic entanglement entropy for general higher derivative gravity, JHEP 01 (2014) 044 [arXiv:1310.5713] [INSPIRE].

[55] J. Camps, Generalized entropy and higher derivative gravity, JHEP 03 (2014) 070 [arXiv: 1310.6659] [INSPIRE].

[56] E. D'Hoker, D.Z. Freedman, S.D. Mathur, A. Matusis and L. Rastelli, Graviton and gauge boson propagators in AdS $S_{d+1}$, Nucl. Phys. B 562 (1999) 330 [hep-th/9902042] [INSPIRE].

[57] M.M. Wilde, A. Winter and D. Yang, Strong converse for the classical capacity of entanglement-breaking and Hadamard channels via a sandwiched Rényi relative entropy, Commun. Math. Phys. 331 (2014) 593 [inSPIRE].

[58] M. Müller-Lennert, F. Dupuis, O. Szehr, S. Fehr and M. Tomamichel, On quantum Rényi entropies: a new generalization and some properties, J. Math. Phys. 54 (2013) 122203 [arXiv: 1306.3142].

[59] R.L. Frank and E.H. Lieb, Monotonicity of a relative Rényi entropy, J. Math. Phys. 54 (2013) 122201 [arXiv:1306.5358].

[60] N. Lashkari, Relative entropies in conformal field theory, Phys. Rev. Lett. 113 (2014) 051602 [arXiv: 1404.3216] [INSPIRE]. 